\title{
Ewing's Sarcoma and Primary Osseous Lymphoma: Spectrum of Imaging Appearances
}

\author{
Marc-André Weber, MD, MSc ${ }^{1}$ Olympia Papakonstantinou, MD \\ ${ }^{1}$ Institute of Diagnostic and Interventional Radiology, University \\ Medical Center Rostock, Rostock, Germany \\ 2 Second Department of Radiology, National and Kapodistrian \\ University of Athens "Attikon" Hospital, Athens, Greece \\ ${ }^{3}$ Department of Radiology, University Surgical Clinic "St. Naum \\ Ohridski," University "Ss. Cyril and Methodius," Skopje, Macedonia \\ ${ }^{4}$ Department of Radiology, AZ Sint-Maarten Mechelen, University \\ Hospital Antwerp, Ghent University, Mechelen, Belgium
}

Violeta Vasilevska Nikodinovska, MD, PhD ${ }^{3}$ Filip M. Vanhoenacker, MD, PhD ${ }^{4}$

\begin{abstract}
Address for correspondence Marc-André Weber, MD, MSc, Institute of Diagnostic and Interventional Radiology, University Medical Center Rostock, Ernst-Heydemann-Str. 6, 18057 Rostock, Germany (e-mail: marc-andre.weber@med.uni-rostock.de).
\end{abstract}

Semin Musculoskelet Radiol 2019;23:36-57.

\begin{abstract}
Keywords

- Ewing's Sarcoma

- primary osseous lymphoma

- radiography

- magnetic resonance imaging

- review

Ewing's sarcoma (ES) is a rare, highly malignant anaplastic stem cell tumor. Histologically, the tumor consists of uniform densely packed small monomorphic cells with round nuclei. The typical appearance at hematoxylin and eosin (H\&E) staining is small blue round cells without any matrix formation. On conventional radiography, ES typically presents as a permeative lesion in the diaphysis of a long bone in a child. A large soft tissue component is another characteristic feature, best depicted by magnetic resonance imaging.

Primary osseous lymphomas are most commonly highly malignant B-cell lymphomas. At $\mathrm{H} \& \mathrm{E}$ histologic staining, the tumor stroma consists of diffuse round-cell infiltrates that resembles the appearance of ES. Although there is no typical imaging appearance of an osseous lymphoma, it should be considered in an adult presenting with a Lodwick grade II or III lesion in the metaphysis or diaphysis of a large long bone, the pelvis, or the vertebral column. Histologic confirmation is mandatory.
\end{abstract}

\section{Ewing's Sarcoma}

\section{Definition}

Ewing's sarcoma (ES) is a rare, highly malignant anaplastic stem cell tumor of neuroectodermal origin, also classified as round-cell sarcoma, to which the primitive neuroectodermal tumors also belong. It mainly arises from the bone. ${ }^{1}$

\section{Demography}

ES represents $\sim 7$ to $10 \%$ of all malignant bone tumors and affects mainly children and adolescents. The mean age is 15 years, and the age peak is 9 to 25 years. However, rarely children and elderly people may be affected. In a series of 64 patients, patient ages ranged between 7 and 67 years. ${ }^{2}$ In Germany, the annual incidence is 120 new cases in a general population of 80 million. Boys are 1.5 to 2.4 times more often affected than girls. ${ }^{2}$ In children, ES is the second most common primary malignant bone tumor after osteosarcoma.

\section{Clinical Manifestations}

Symptoms consist of nonspecific, non-exercise-related pain in the area of the tumor and swelling. General symptoms such as fever, fatigue, loss of appetite, and weight may appear more frequently than in other malignant bone tumors. ${ }^{1}$

\section{Localization}

ES mainly is localized in the long bones of the extremities and most commonly within the diaphysis close to the metaphysis. The most commonly affected bone is the femur (25\%)(-Fig. 1), followed by the tibia (11\%)( - Fig. 2 ), humerus (11\%), and pelvic
Issue Theme Bone and Soft Tissue Tumors; Guest Editors, Marc-André Weber, MD, MSc and Radhesh Lalam, MBBS, MRCS, FRCR
Copyright $\odot 2019$ by Thieme Medical Publishers, Inc., 333 Seventh Avenue, New York, NY 10001, USA. Tel: +1(212) 584-4662.
DOI https://doi.org/ $10.1055 / \mathrm{s}-0038-1676125$. ISSN 1089-7860. 

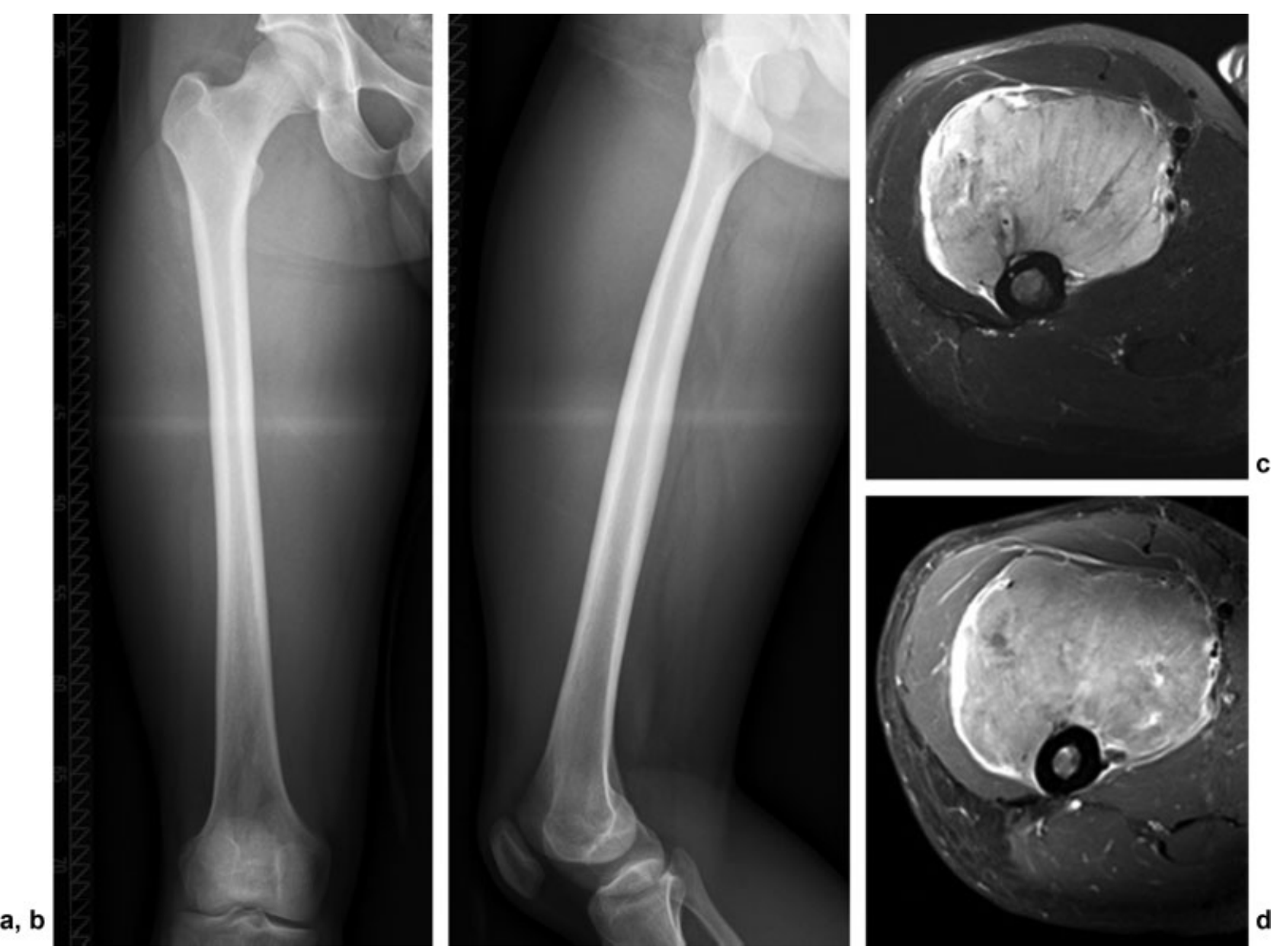

Fig. 1 Extraskeletal Ewing's sarcoma in a 26-year-old man. (a) Anteroposterior projection and (b) lateral projection radiographs performed after a swelling and increasing painfulness of his right thigh that he had observed for 5 days. No bony erosion is visible, but an increased opacification of the quadriceps femoris muscle is observed. (c, d) MRI axial T2-weighted fat-saturated sequences demonstrate the large soft tissue tumor predominantly within the vastus intermedius muscle. The patient was included in the EURO-EWING 2008 study.

bones (11\%) (-Fig. 3). ${ }^{1}$ Other localizations are the fibula (7\%) (-Figs. 4 and $\mathbf{5}$ ) and ribs (6\%) (-Fig. 5). ES can virtually involve all bones of the body and may also involve extraskeletal sites (-Fig. 1). An isolated involvement of the soft tissue (extraskeletal ES) was observed in $1.5 \%$ of all cases in a series of 64 patients. ${ }^{2}$ Compared with osteosarcoma, flat bones are more often affected in ES. Rarely, a bicentric ES or synchronous involvement of multiple bones may be seen ( - Fig. 6 ), whereas transarticular spread can rarely be seen in sacroiliac joints. ${ }^{3} \mathrm{Up}$ to $15 \%$ of all ES cases are localized in the vertebral column, with the lumbar spine and the sacrum as the most commonly affected; the location of ES tends to follow the distribution of red marrow. The mean age at initial clinical presentation is 19.3 years, slightly higher than the mean age of ES of the extremity bones. ${ }^{4}$ Metastases of ES affecting the vertebral column are more common than a primary ES of the spine. ${ }^{5}$

\section{Pathology}

Macroscopically, the tumor has the aspect of fish flesh. At histology, the tumor consists of uniform densely packed small monomorphic cells with round nuclei that are generally larger than in primary osseous lymphoma. The typical appearance at hematoxylin and eosin (H\&E) staining is small blue round cells without any matrix formation ( - Fig. 2). At immunostaining, an expression of the cellular surface antigen CD99, a product of the MIC-2 gene, is characteristic. The characteristic reciprocal translocation between chromosome 11 and $22(\mathrm{t}[11 ; 22])$, which affects in $85 \%$ the EWS and FLI1 gene, can be confirmed by using break- apart fluorescence in situ hybridization (- Fig. 2). ${ }^{1,6,7}$

\section{Imaging}

Conventional radiography (CR) reveals an aggressive osteolytic lesion with a wide zone of transition and with a moth-eaten or permeative pattern in most cases. ${ }^{8}$ In a series of 64 patients, most tumors were mixed lytic-sclerotic (in 75\%) and purely lytic in $25 \% .^{2}$ Complex periosteal reactions like onion-skinned, spiculae, "sunburst," or Codman's triangle can be seen (- Fig. 5) in 27 to $50 \%$ of all cases. ${ }^{2}$ Onion-skinned or multilamellated periosteal reactions may be encountered in $25 \%$ of cases. Computed tomography (CT) is more sensitive than CR to detect bony destruction in anatomically complex regions such as the vertebral column and the pelvic bones due to the lack of superimposition. In flat bones such as the pelvic bones, ES may have a predominantly sclerotic appearance ( - Fig. 3 ) that overall represents $\sim 10 \%$ of cases. The reason for the sclerotic 
appearance is mainly necrotic bony changes because tumorrelated formation of new bone is not a histologic feature of ES. ${ }^{1}$

Magnetic resonance imaging (MRI) is the method of choice for local staging because it demonstrates the true tumor extension (-Figs. 4, 5, 6, 7). The MRI protocol has to cover the entire tumor extent as well as the adjacent joints and the entire bone where the tumor originates because skip lesions

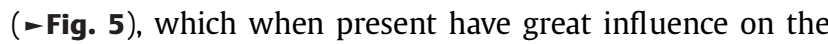
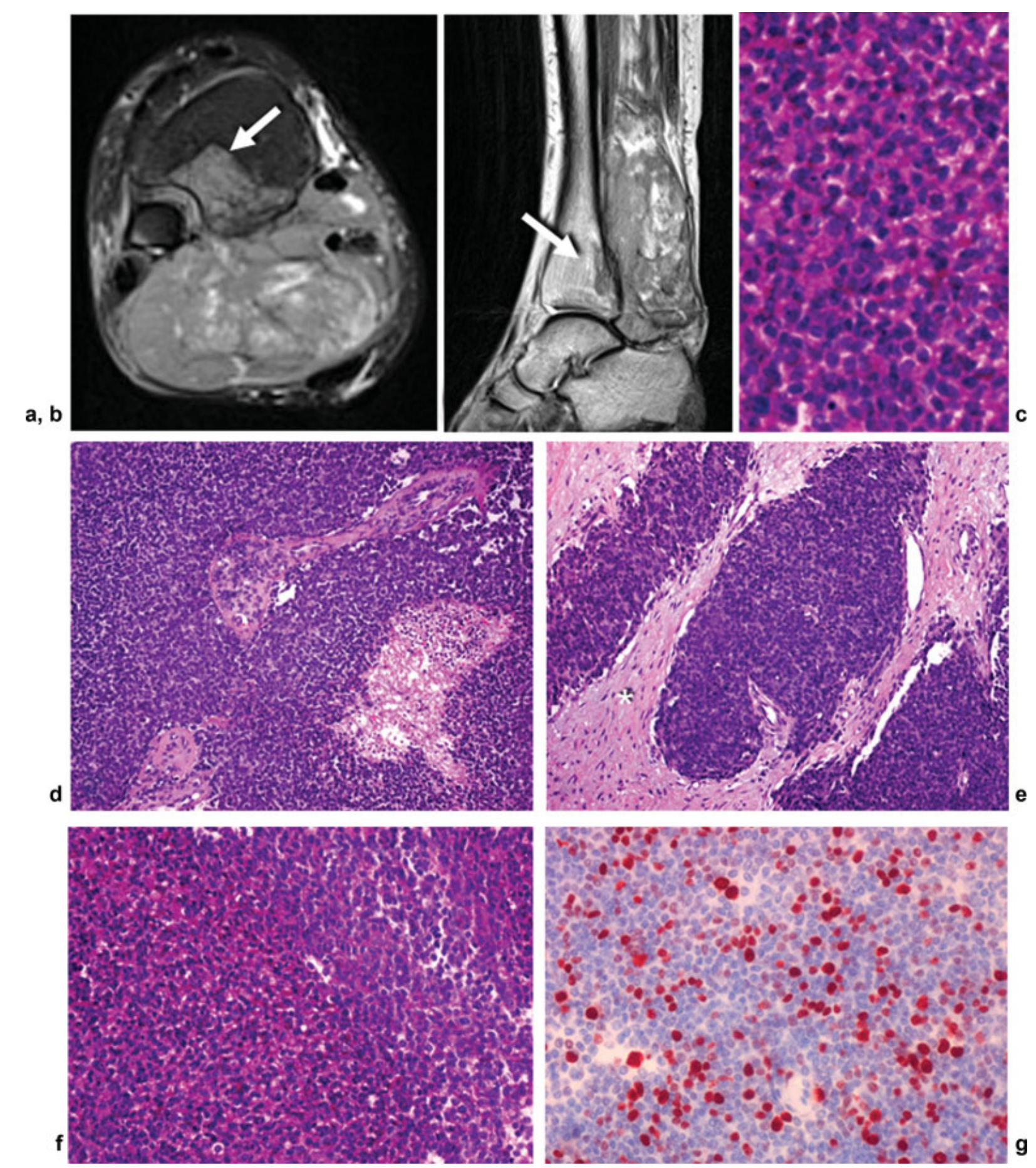

Fig. 2 Predominantly extraosseous Ewing's sarcoma (ES) with histopathologic correlation in a 25-year-old woman. (a) MRI axial T2-weighted fat-saturated sequence and (b) sagittal T2-weighted sequence illustrate the infiltration of the dorsal tibia (arrows) and the large soft tissue mass ventral to the Achilles tendon. (c) Hematoxylin and eosin (H\&E) staining demonstrates monomorphous cells with a relatively small eosinophilic cytoplasm. (d, e) The H\&E-stained section with a lesser magnification demonstrates that ES stroma consist mainly of infiltrates of small round blue cells with small amounts of relatively cell-poor connective tissue (asterisk). (f) The slightly higher magnification better illustrates the monomorphous small round blue cells typical of ES in hematoxylin-eosin staining. (g) The MIB-1 immunostaining of Ki-67 illustrates mitotic activity of 20\%. (h) There was a homogeneous and strong membranous expression of CD99, a p30/32 surface antigen, in the CD99 immunostaining. (i) The diagnosis of ES could be confirmed by using break-apart EWS fluorescence in situ hybridization analysis with the EWS gene locus encompassing DNA probes combination. Tumor cell nuclei show besides an orthologous fused another dislocated hybridization pair (arrow) confirming the translocation $\mathrm{t}(11 ; 22)$ of the EWS gene locus. 


\section{h}
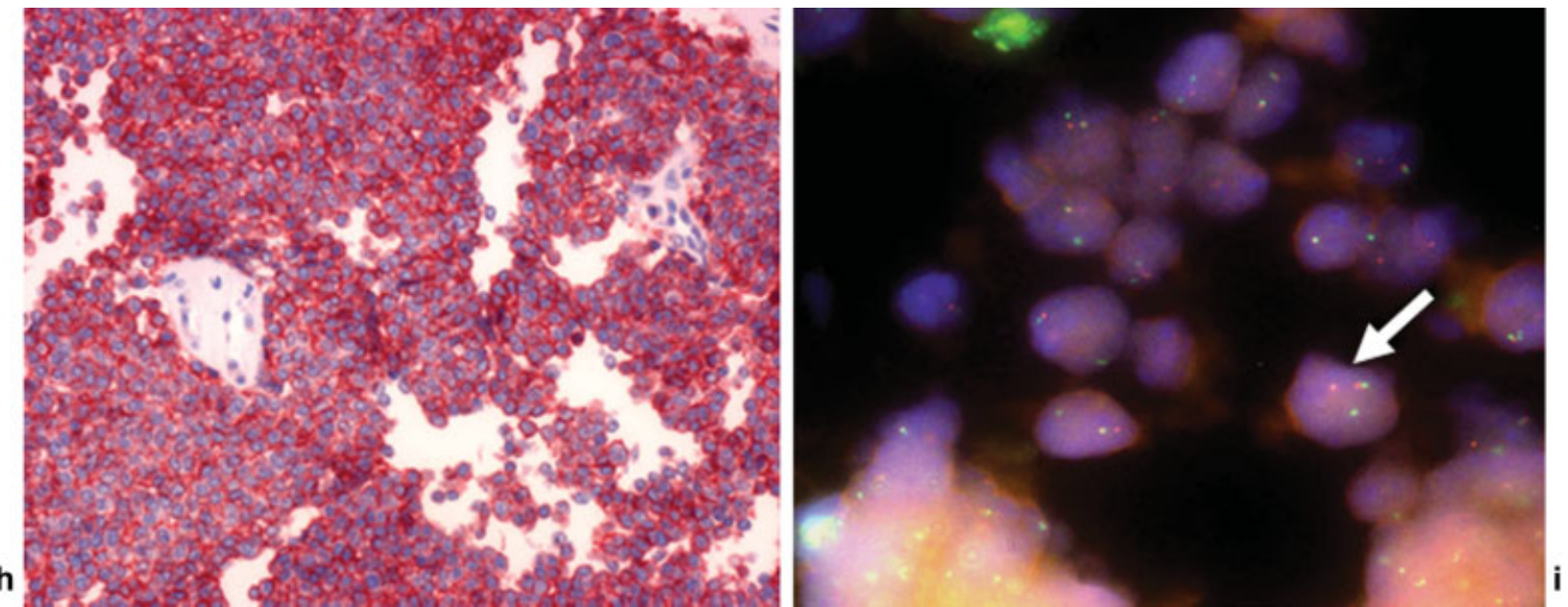

Fig. 2 (Continued)
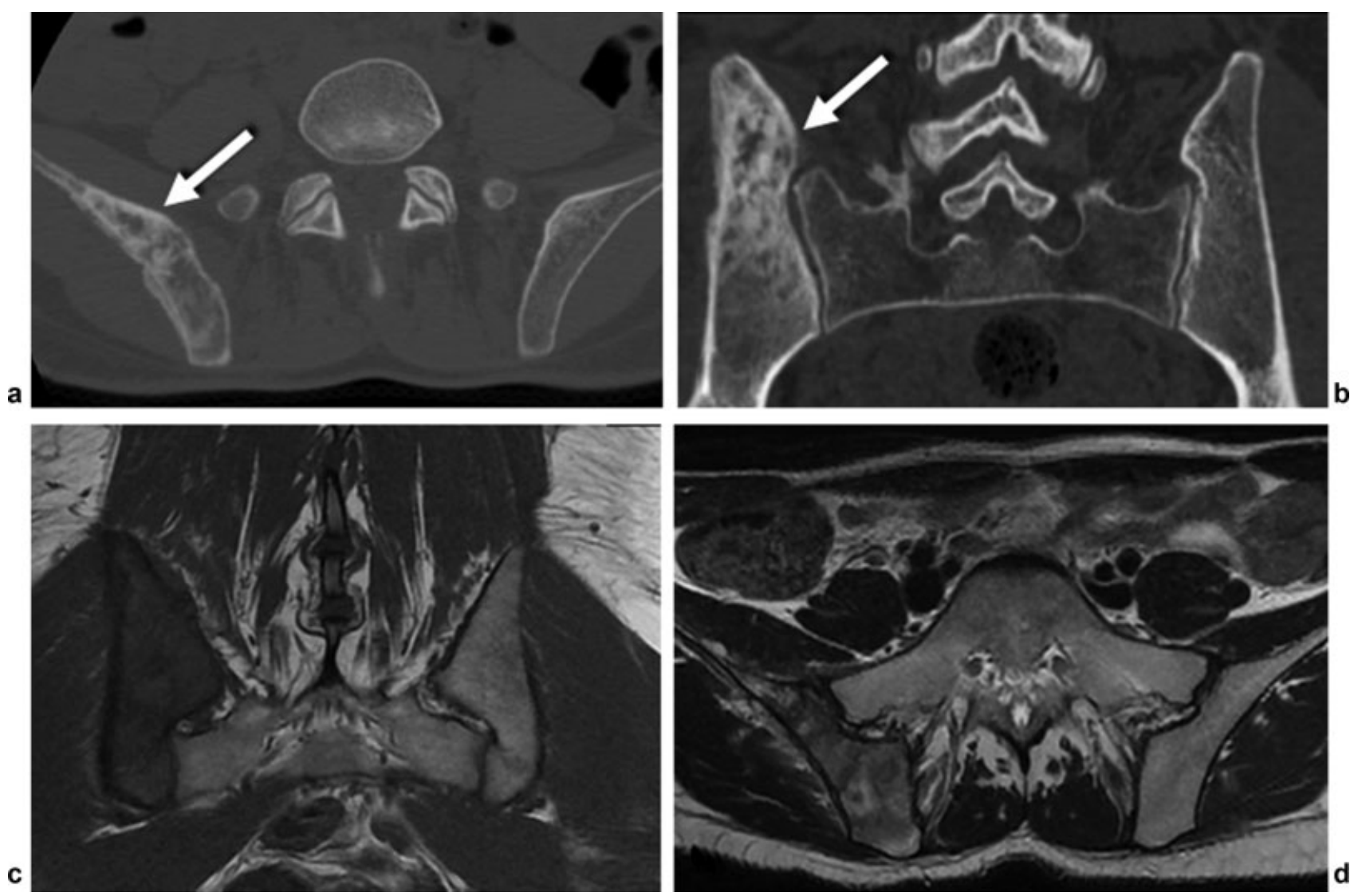

Fig. 3 Predominantly sclerotic Ewing's sarcoma (ES) of the right os ilium (arrows) in a 26-year-old woman that was subsequently treated by polychemotherapy and radiotherapy. Computed tomography in (a) axial and (b) coronal plane. (c) MRI using a coronal T1-weighted and (d) an axial T2-weighted sequence. These predominantly sclerotic types of ES are not uncommon within the pelvis.

treatment planning, are frequent. Skip metastases at initial presentation were present in $14 \%$ of all cases in a series of 64 patients. $^{2}$ The signal intensity of the tumor tissue of the ES is hypointense on T1-weighted images and hyperintense on T2weighted images. A large soft tissue component is highly characteristic (96\% of all cases), ${ }^{8}$ showing marked contrast enhancement. ${ }^{2}$ Focal areas of cortical destruction are frequent (92\% of all cases), ${ }^{8}$ allowing continuity between the intraoss- eous and extraosseous components (-Figs. 5, 6, 7). This continuity is also commonly seen as subtle channels extending through the cortex at MRI, a finding that reflects the underlying pathologic appearance ${ }^{8}$ because the communication between the medullary canal and soft tissue components may be through focal cortical destruction or more commonly through permeation of the cortical haversian canal system and along neurovascular channels with small nests of tumor cells. ${ }^{8}$ 

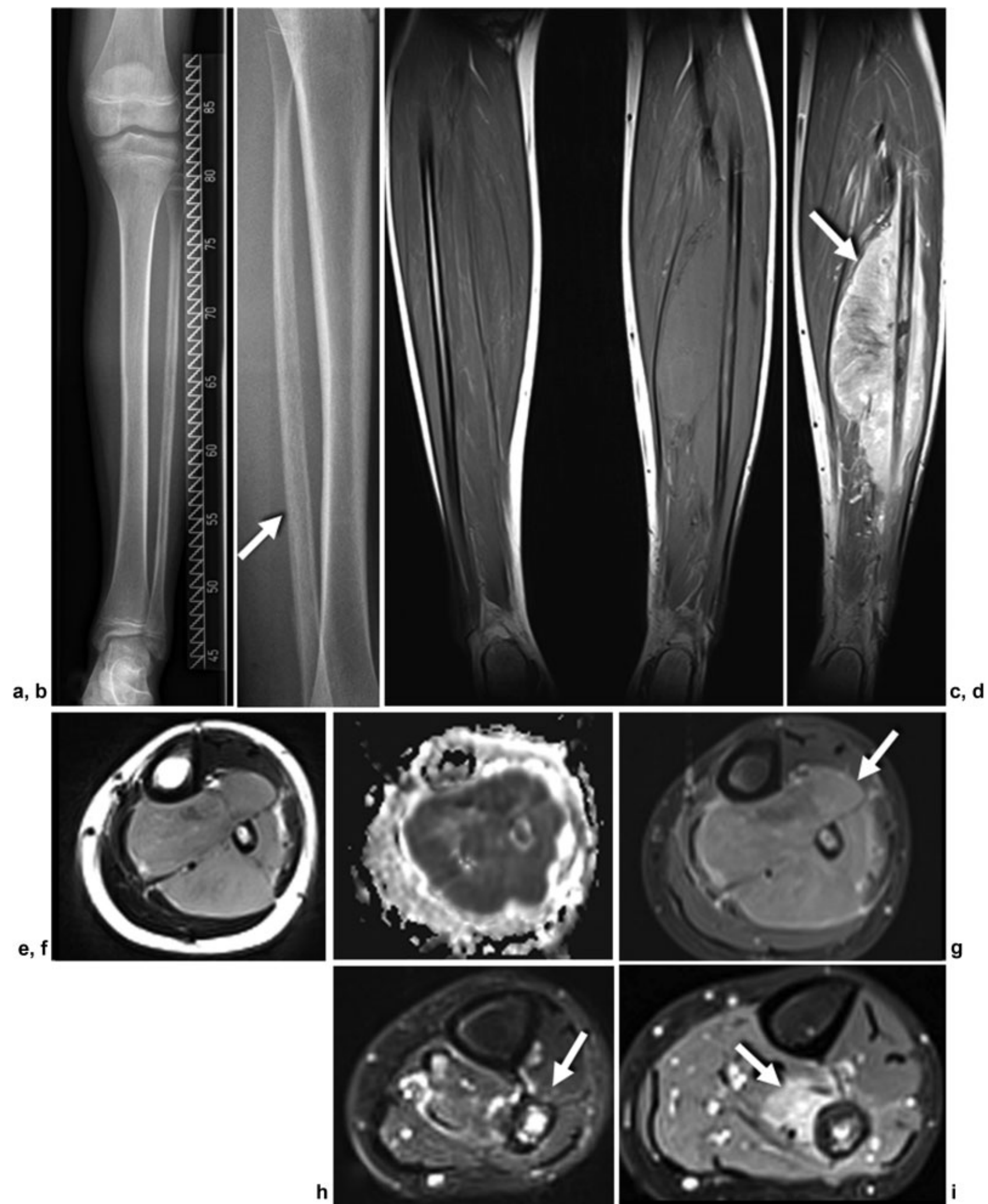

Fig. 4 Ewing's sarcoma in a 12-year-old boy. Projection radiography in (a) anteroposterior and (b) lateral view demonstrate only a blurred cortical bone of the diaphysis of the fibula (arrow). (c) MRI coronal T1-weighted, (d) coronal contrast-enhanced T1-weighted, (e) axial T2-weighted, (f) axial apparent diffusion coefficient (ADC) map, and ( $($ ) axial contrast-enhanced fat-saturated T1-weighted sequence illustrate the large soft tissue component surrounding the fibula (arrows). (f) The tumor's stroma consisting of densely packed cells (at histology small round blue cells with characteristic proof of EWSR1-ERC fusion is reflected by the low ADC values of the tumor tissue). Six months after neoadjuvant polychemotherapy, the tumor mass has substantially decreased as evidenced by MRI (arrows): (h) axial fat-saturated T2-weighted, (i) axial contrast-enhanced fat-saturated T1-weighted, (j) coronal short tau inversion recovery, (k) coronal T1-weighted, and (I) sagittal T2-weighted sequences. (m) FDG-PET/CT shows slight tracer uptake within the left fibula but no metastases. (n) After partial resection of the tumor-bearing bone and complete resection of the tumor including the soft tissue component, therapy proceeded with chemotherapy and radiotherapy of the lower leg. 


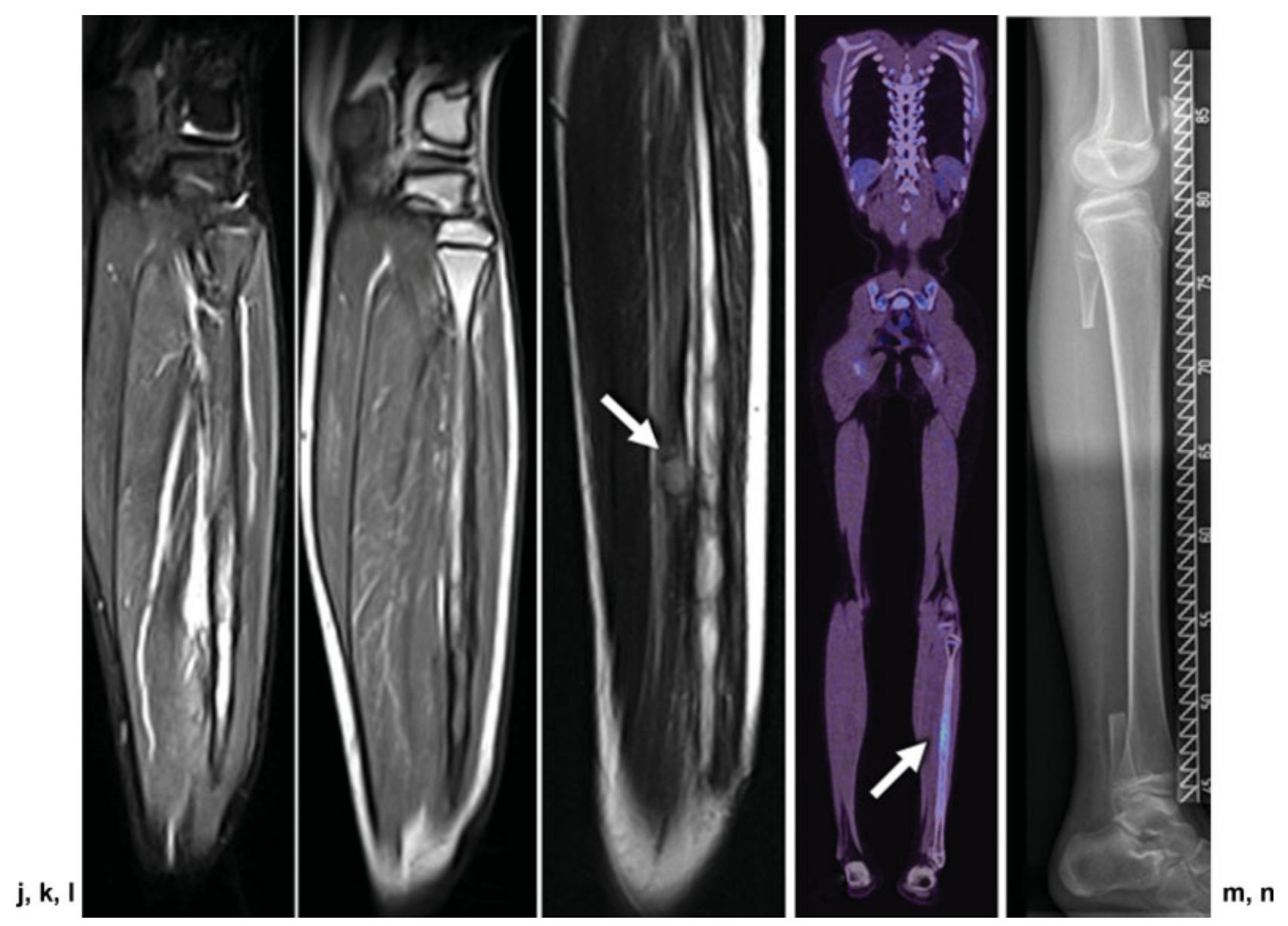

Fig. 4 (Continued)

Extraskeletal ES commonly demonstrates a nonspecific radiologic appearance of a large soft tissue mass affecting the paraspinal region or lower extremity, ${ }^{8}$ and it may be occult on CR (-Fig. 1). Another imaging characteristic that may occur in ES as well as osseous lymphoma is the so-called wraparound sign. This means that the cortex at T2-weighted images appears regularly hypointense, although there is a large soft tissue component that appears to be wrapped around the bone. This is believed to be due to the permeative growth of the tumor so that the calcified bone is not destroyed to a major extent (-Figs. 4, 5, 6, 7).

Because ES most often metastasizes hematogenously into the lung (although lymphatic and osseous spread may also occur but is rare; the incidence of regional node involvement is $\sim 3 \%$ in skeletal $\mathrm{ES}^{9}$ ), staging consists of CT of the thorax and abdomen as well as bone scintigraphy or positron emission tomography (PET) using fluorodeoxyglucose (FDG) as a tracer to detect or exclude metastases. In $25 \%$ of all cases, there are metastases present at the time of diagnosis of ES. According to the EWING 2008 study, ${ }^{10}$ initial imaging of ES consists of MRI for local staging, chest CT, whole-body scintigraphy, and FDG-PET. After completion of imaging, the final diagnosis will be verified at histology and by genetic markers from biopsy material. Biopsy should be performed in a dedicated sarcoma treatment center. ${ }^{11}$

\section{Differential Diagnosis}

In general, all tumors consisting of small round blue cells may have similar imaging features. The main differential diagnoses consist of osteolytic osteosarcoma and small-cell osteosarcoma in children and adolescents, osteomyelitis in all age groups, and non-Hodgkin's lymphoma in adulthood. ${ }^{12}$ In children, unifocal Langerhans cell histiocytosis is another differential, but the periosteal reaction is often less aggressive, and an eosinophilic granuloma may have a sequestrum. In the spine, ES may mimic spondylitis because of its high signal intensity in T2-weighted images, ${ }^{4,13}$ whereas in the rare case of sacroiliac joint involvement, it should be distinguished from septic sacroiliitis (-Fig. 7). ${ }^{3}$

\section{Therapy and Treatment Monitoring}

Treatment is interdisciplinary and consists of induction chemotherapy and local therapy with subsequent chemotherapy and/or radiation therapy within multicentric study protocols. ${ }^{14,15}$ Such multicentric study protocols are, for instance, the EURO-EWING 99 (six cycles of induction chemotherapy followed by local therapy, and adjuvant chemotherapy using different regimens according to the individual patient's risk factors) or the EURO-EWING 2008 study (- Figs. 4 and 6). ${ }^{16,17}$ For the latter, neoadjuvant chemotherapy with histopathologic assessment of tumor response to induction neoadjuvant 
chemotherapy, followed by local therapy like surgical tumor resection and reconstruction, and optionally followed by radiation therapy is recommended. Radiation therapy alone as local therapy is the therapy of choice whenever surgery is not possible but has the drawback of a higher local recurrence rate. A second chemotherapy phase as adjuvant chemotherapy is followed in a risk-adapted way. The success of neoadjuvant chemotherapy is assessed locally by using CR and MRI and FDG-PET (according to EURO-EWING 2008) to assess therapy response and local recurrence (-Fig. 4).

$\mathbf{a}, \mathbf{b}$
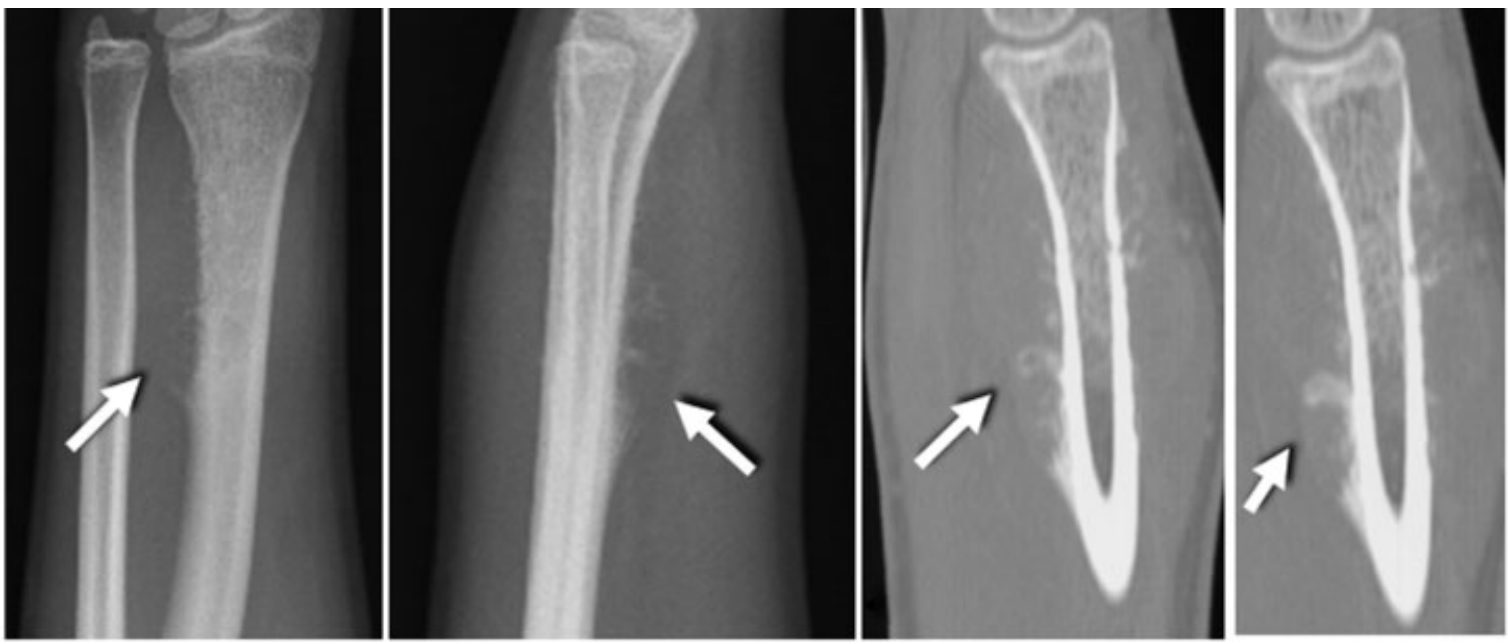

c, d
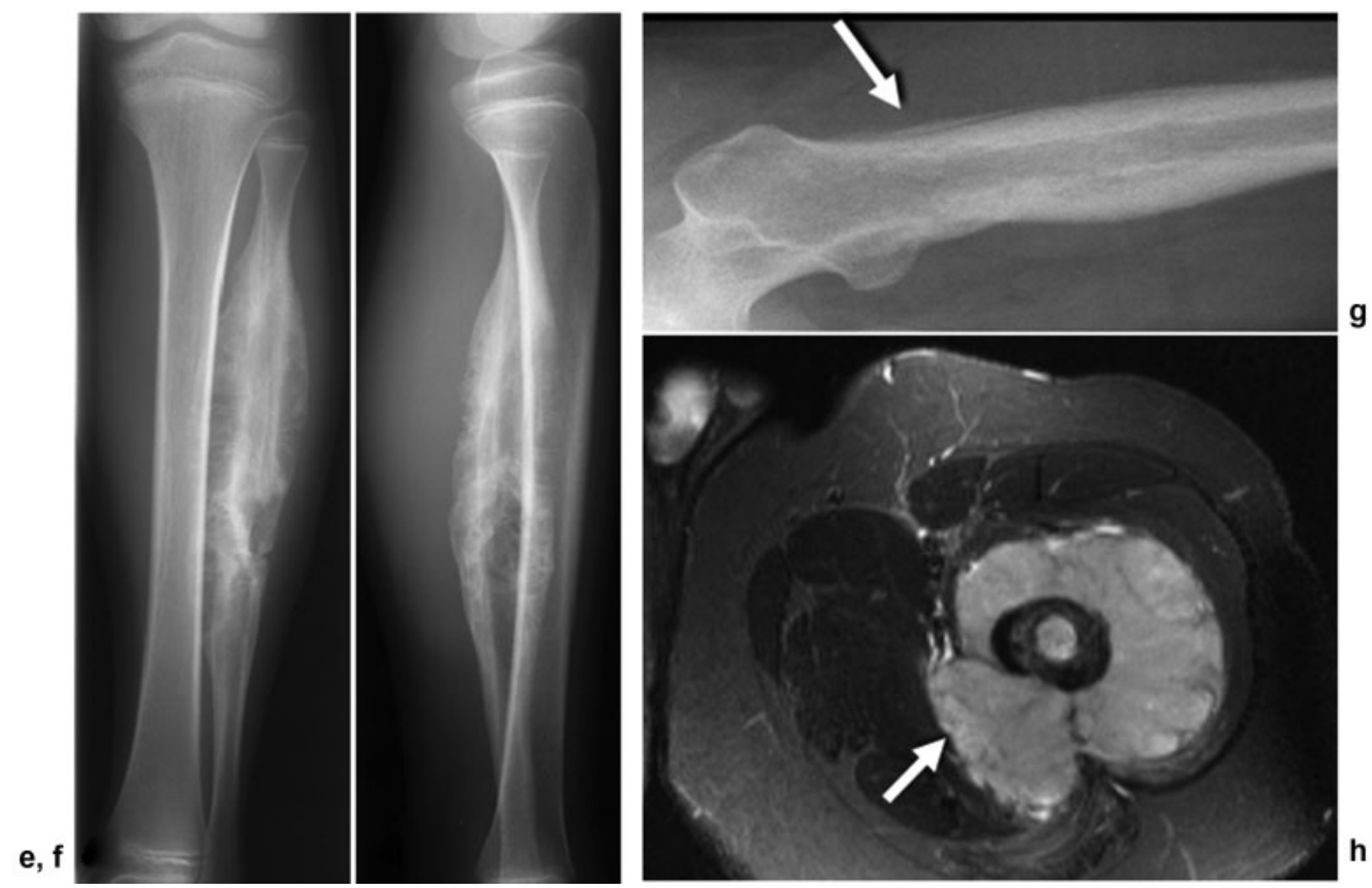

Fig. 5 Gallery of Ewing's sarcoma (ES) images. Projection radiographs in (a) anteroposterior (AP) and (b) lateral view as well as sagittal reformatted CT (c, d) of ES of the diaphysis of the left radius in a 14-year-old girl (arrows). Note a hair-on-end periosteal reaction at the ulnar and palmar aspect of the diaphysis of the radius on plain radiographs. On CT, the extent of the periosteal reaction is more obvious and shows that both the palmar and dorsal side of the radius are involved. Note also focal irregular delineation of the dorsal cortex of the radius. Projection radiographs in (e) AP and (f) lateral view of ES of the left fibula in an 11year-old boy. There is fusiform bone expansion of the diaphysis of the fibula with a distinct periosteal reaction with a variable pattern, consisting of partially onion-skinned and partially perpendicular (sunburst pattern) to the underlying cortex. The lesion has a mixed appearance with areas of osteolysis, cortical destruction, and sclerotic areas. ES of the left femur with onion-skin periosteal reaction (arrow), fusiform bone expansion of the diaphysis, and ill-defined osteolysis with cortex permeation on (g) projection radiography, (h) large soft tissue component (arrow) and wraparound sign on axial T2-weighted fatsuppressed MRI, and (i) skip lesion (arrow) in the femur diaphysis depicted by unenhanced coronal T1-weighted MRI. ES of the left lower thoracic rib cage in a 12year-old boy. There is cortical destruction of the rib with large associated soft tissue mass extending in the pleura, left paravertebral muscles and left neuroforamen. The lesion is inhomogeneous on T2-weighted image with fat suppression with areas of low to intermediate signal intensity and hyperintense areas. There is vivid enhancement of the lesion; ( $\mathbf{j})$ sagittal T2-weighted, (k) axial T1-weighted, and (I) contrast-enhanced fat-saturated T1-weighted sequences. 

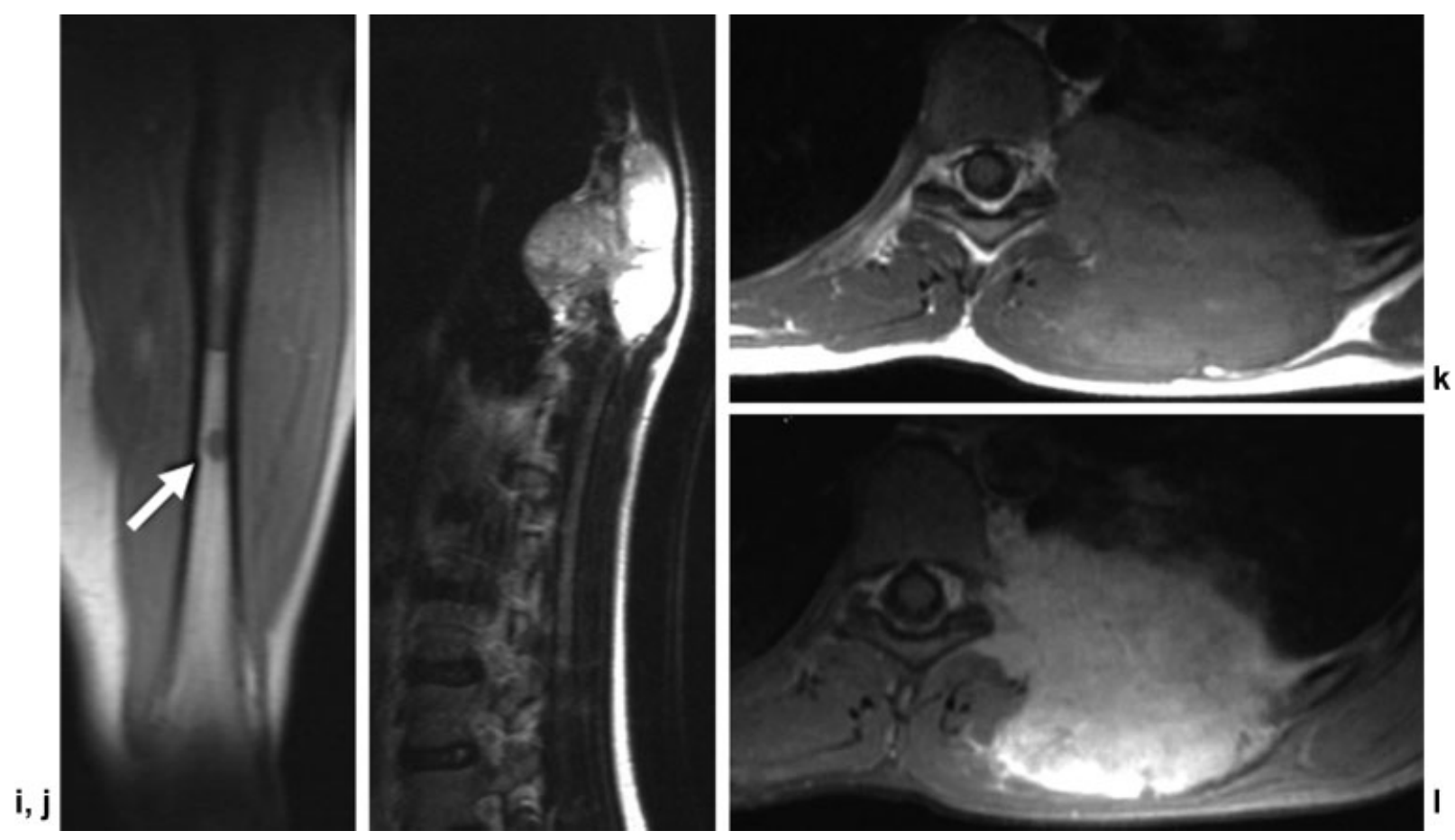

Fig. 5 (Continued)

Dynamic contrast-enhanced MRI may help differentiate residual or recurrent tumor from posttherapeutic changes because tumor tissue enhances early and more rapidly during the first pass of contrast, whereas reactive tissue resulting from posttherapeutic changes enhances later and more slowly. ${ }^{18}$ Low-dose CT of the chest is recommended in adults for pulmonary follow-up; in children a chest radiograph may be enough. Most relapses occur in the first 3 years of follow-up; late relapses have rarely been observed even after $\geq 15$ years. Follow-up intervals should be 2 to 3 months during the first 3 years, 6 months until 5 years, and at least once yearly thereafter according to the clinical recommendations of the European Society for Medical Oncology. ${ }^{19}$ Overall, the 5 -year-survival rate is 64\% (up to $75 \%$ in those patients who present without known metastases). ${ }^{20,21}$ Age, tumor volume, and extent of metastatic spread are relevant risk factors. ${ }^{15}$

\section{Primary Osseous Lymphoma}

\section{Definition}

Lymphomas are a heterogeneous group of primary neoplasms of the lymphoid tissue. Malignant lymphocytes normally accumulate in the lymph nodes, causing lymph node disease. Occasionally they may spread to the blood (leukemic phase) or infiltrate organs outside the lymphoid tissue. Lymphomas may be classified as Hodgkin's disease or nonHodgkin's lymphoma. Among all cases of lymphoma, 40 to $50 \%$ involve the skeletal system, generally from metastatic disease. Non-Hodgkin's lymphoma affects bones more commonly than Hodgkin's lymphoma, either as primary or secondary. Hodgkin's lymphoma, characterized by invol- vement of Reed-Sternberg cells, most commonly presents with progressive painless enlargement of peripheral lymph nodes, especially around the cervical region. At the time of diagnosis, osseous involvement is uncommon, and even in the late stages only 9 to $35 \%$ of cases have any bony involvement. Rarely Hodgkin's lymphoma presents as an osseous lesion without involvement of lymph nodes. ${ }^{22}$ Primary osseous lymphoma (primary lymphoma of bone [PLB]) has to be distinguished from a secondary bone involvement by lymphomas arising from visceral organs or lymph nodes. The primary non-Hodgkin's lymphoma of the bone is an aggressively growing tumor consisting of malignant lymphatic cells that affects a single bone with or without regional lymphadenopathy. The polyostotic PBL is defined as multifocal but exclusive involvement of the skeleton, whereas the disseminated lymphoma affects various organs with or without secondary involvement of the skeleton. ${ }^{23}$ Thus for the correct categorization of an osseous lymphoma, the radiologist's role is to exclude simultaneous extraosseous manifestations of lymphoma at other localizations. Primary osseous lymphomas are most commonly highly malignant Bcell lymphomas. ${ }^{24}$

\section{Demography}

PLB is an uncommon clinical entity and a rare presentation of non-Hodgkin's lymphoma. It can occur at any age, but it has a wide age peak between 30 and 60 years. ${ }^{24,25}$ An overall median age of 45 years (range: 7-87 years) was reported in a study on 119 patients with a female-to-male ratio of $1: 1.53 .{ }^{25}$ PLBs represent 3 to $5 \%$ of all malignant bone tumors, 4 to $5 \%$ of extranodal lymphoma, and $<1 \%$ of all nonHodgkin's lymphoma. ${ }^{26}$ Diffuse large B-cell lymphoma 
(DLBCL) accounts for most cases of primary osseous lymphomas. DLBCL usually associates with single bone involvement, whereas the less frequent types, like B-lymphoblastic lymphoma, are associated with multifocal involvement. Primary osseous lymphoma is uncommon in children and adolescents. $^{27}$

\section{Clinical Manifestations}

The leading symptom is local pain. General symptoms such as fever, night sweats, and fatigue that appear frequently in extraskeletal lymphomas are typically not frequently observed in PLBs.

\section{Localization}

Approximately $50 \%$ of all PLBs are localized within the skeleton of the trunk and skull; the other $50 \%$ are situated in the long bones, especially the femur ( $\mathbf{F i g . ~ 8 ) ; ~ i n ~ g e n e r a l , ~}$ bones containing red marrow are affected. Of the latter, two thirds are localized at the metaphysis and one third at the diaphysis. Epiphyseal involvement is rare. ${ }^{28}$ About $80 \%$ of PLBs are observed unifocally within the bone (-Fig. 9) ${ }^{24}$ In a 2014 study, the femur was the most commonly involved single site in PLBs, whereas those with multifocal bone lesions most frequently presented with spine disease (-Figs. 8 and 10). ${ }^{29}$ Secondary lymphomas also favor the
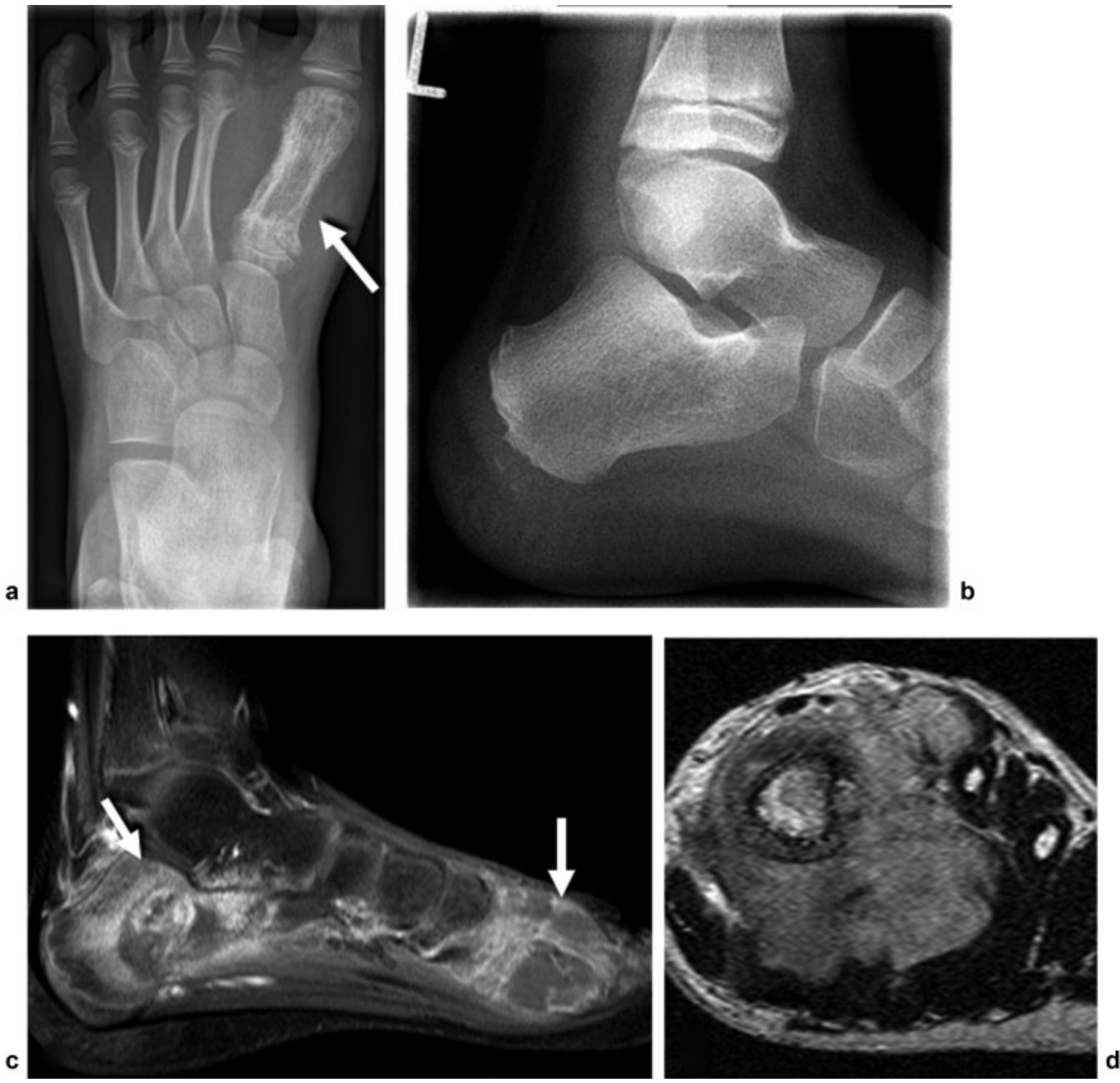

Fig. 6 Rare bicentric Ewing's sarcoma (ES) in a 7-year-old boy. Projection radiography in (a) anteroposterior and (b) lateral projection. Inhomogeneous lytic and sclerotic changes with blurred cancellous and cortical bone and a slight periosteal reaction of the first metatarsal bone are visible (arrows), but no obvious changes in the calcaneus of the left foot are visible. The synchronous manifestation of two ESs within the calcaneus and first metatarsal bone is elucidated by MRI (arrows). (c) Contrast-enhanced fat-saturated sagittal T1-weighted, (d) coronal T2-weighted, (e) axial T1-weighted, and (f) axial short tau inversion recovery sequences. The large soft tissue component encompassing the first metatarsal bone and the cortical bone penetration is also well depicted in (d). (g) Nuclear bone scan and (h) FDG-PET/CT show no other tracer taking up tumors in the rest of the body. Therapy according to the EURO-EWING 2008 study was followed by amputation at the mid lower leg level. (i) The lateral projection radiograph 3 years later shows a normal stump with slight ossifications at the end of the stump. The boy is satisfied with the situation, can participate in sports, and has no pain at all. 
$e, f$
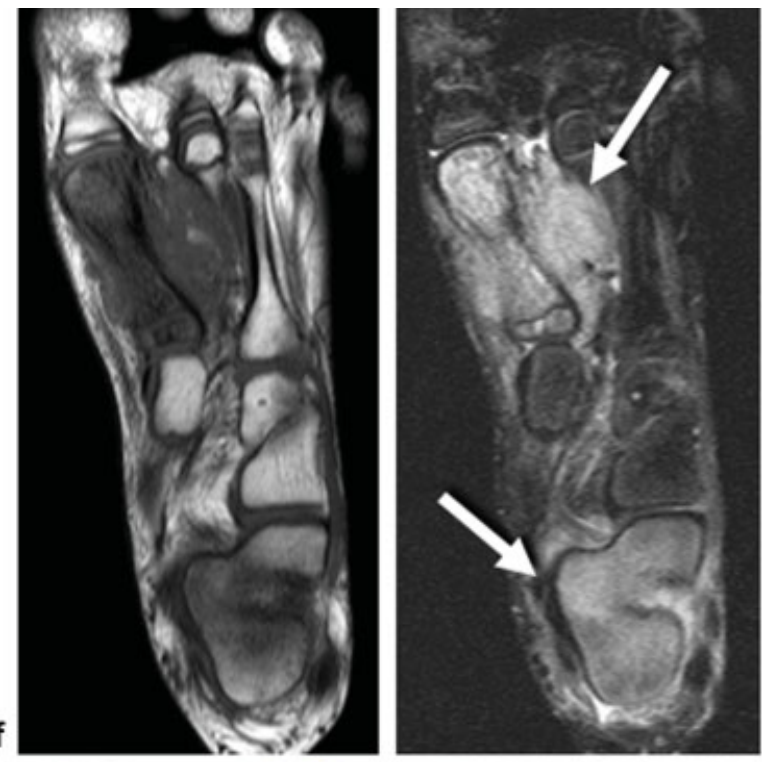

h, i

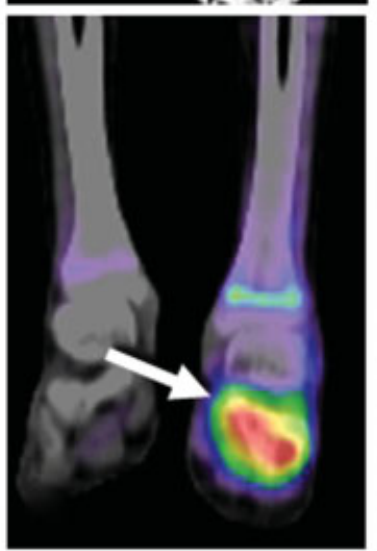

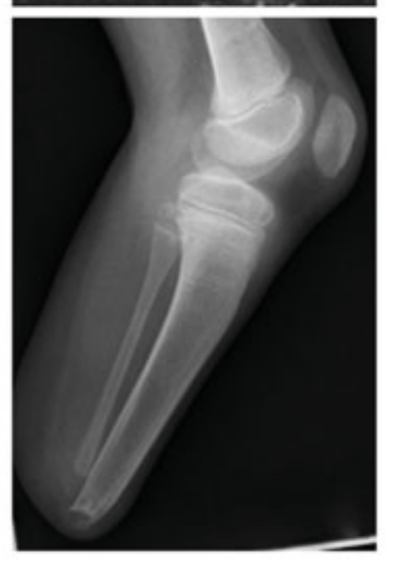
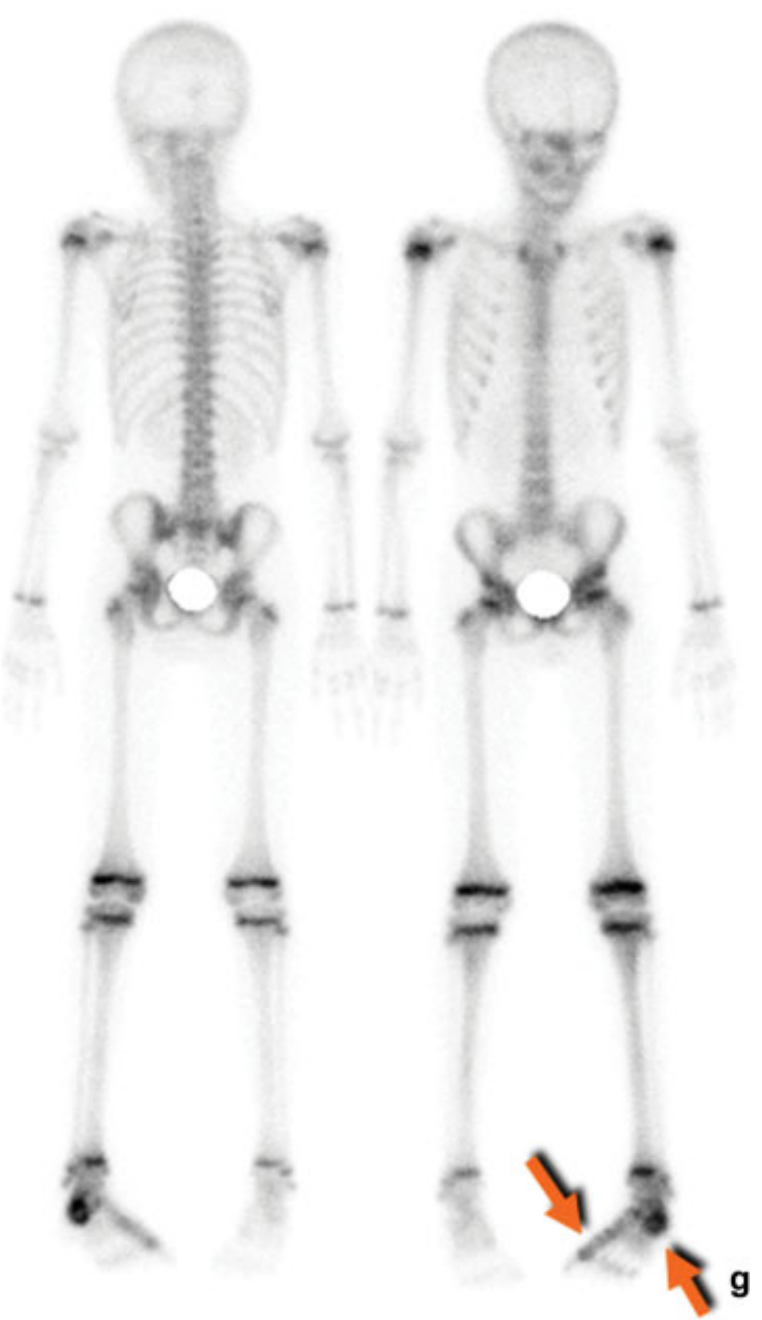

Fig. 6 (Continued)

spine and should be distinguished from primary multifocal lymphoma. ${ }^{30}$

\section{Pathology}

PLBs have an indistinct tumor margin and show an invasive diffuse growth pattern. The bony cortex nearly always is infiltrated and also mostly penetrated. The pattern of extensive marrow disease and surrounding soft tissue masses but without extensive cortical destruction was reported nearly exclusively in round-cell tumors such as PLB, multiple myeloma, and ES. ${ }^{31}$ An explanation for this finding is the spread of tumor cells from the marrow through small vascular channels that run through the cortex into the surrounding soft tissue. ${ }^{31}$ On H\&E histologic staining, the tumor stroma consists of diffuse round-cell infiltrates. This aspect resembles the appearance of ES. The diffuse infiltrates cause secondary bone resorption. At immunohistochemical analysis, nearly all lymphomas express common leukocyte antigen and B-cell markers like CD20 (-Fig. 10). ${ }^{31}$

\section{Imaging}

On CR or CT, PLBs may appear as solitary osteolytic or multiple osteolytic lesions with an aggressive destruction pattern
(-Fig. 9). Similar to ES, the permeative and moth-eaten destructive pattern is an indication of the presence of a tumor consisting of small or round cells. The periosteal reaction, reported in $\sim 60 \%$ of cases, is often aggressive and may be complex. ${ }^{31} \mathrm{CT}$ may be useful to demonstrate bony destruction and, if present, a sclerosing pattern within the spine and pelvis (-Fig. 11). Osteosclerotic changes may be observed as well; however, predominant osteosclerosis is unusual in primary osseous non-Hodgkin's lymphomas. ${ }^{24,32}$ A sequestrum within a lucent lesion occasionally is seen and is optimally visualized on CT, although it is a nonspecific finding. ${ }^{31}$

In the spine, the often multisegmental lesions may appear osteolytic or mixed osteolytic and sclerotic (-Fig. 10). Although osseous lymphoma was classically considered in the differential diagnosis of an ivory vertebra, this is a relatively rare occurrence. ${ }^{33,34}$ The mixed sclerotic-lytic type is more common in secondary lymphomas and after treatment. ${ }^{30}$ However, radiographic findings may be very subtle even in the presence of aggressive appearance on other imaging modalities (- Fig. 12). ${ }^{31,35}$ MRI, similar to ES, is the method of choice for the work-up of symptomatic areas and especially in the case of suspected compression of the spinal cord or 
a
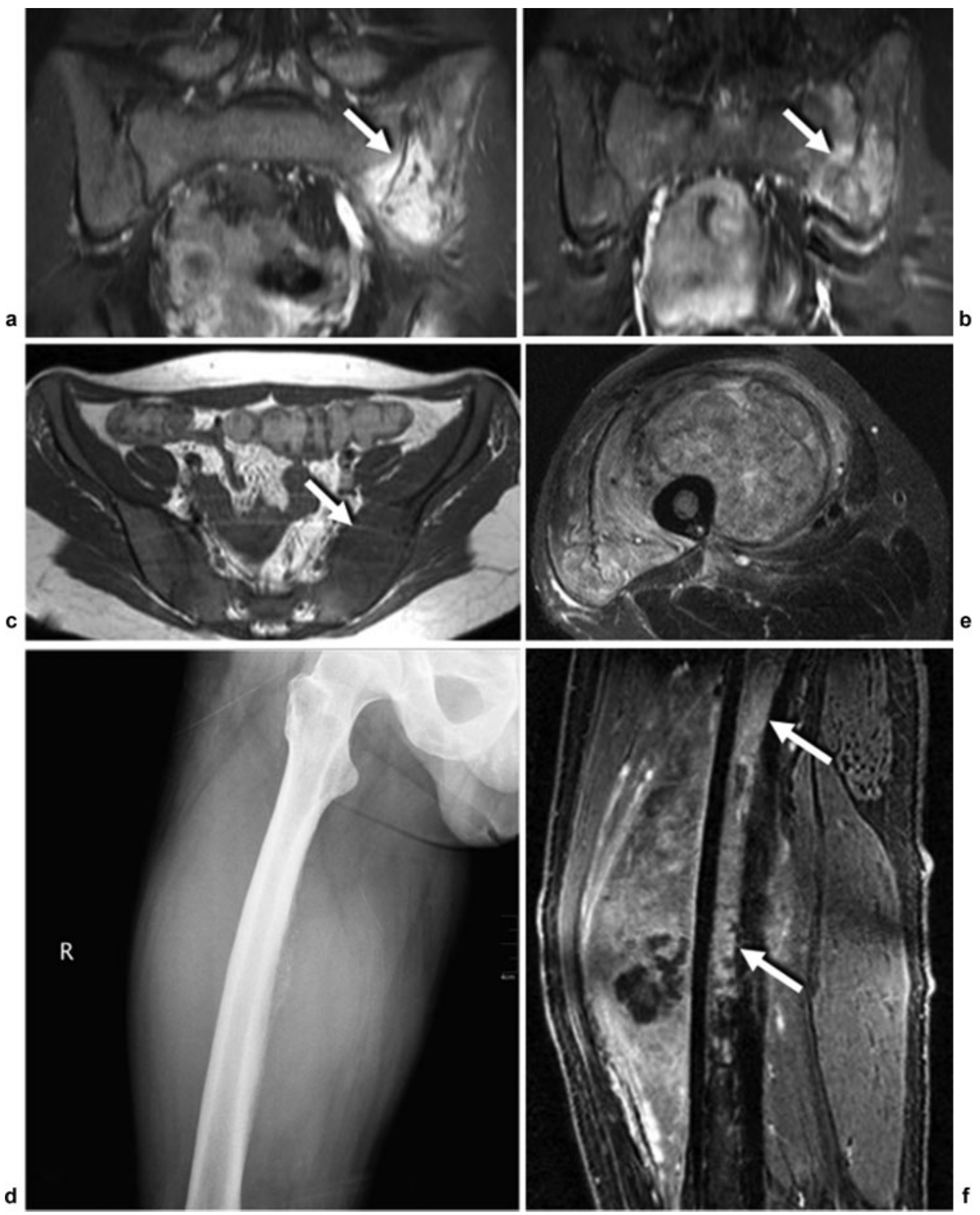

Fig. 7 Gallery of Ewing's sarcoma (ES) images. (a-c) An 18-year-old woman with ES and involvement of the left sacroiliac joint (arrows), presented with low back pain, fever, and malaise. Initial MRI shows transarticular infiltration of the left sacroiliac joint. In conjunction with clinical symptoms, the findings were misinterpreted as osteomyelitis, and the patient received antibiotic treatment for 2 months without any clinical improvement. (a) Coronal fat-saturated proton-density-weighted image, (b) coronal fat-saturated contrast-enhanced T1-weighted image, and (c) axial T1-weighted image. (d-f) A 37-year-old man with ES presented with painful swelling. (d) Projection radiograph in anteroposterior view shows cortical destruction and periosteal reaction at the upper third of the femoral diaphysis. (e) Axial fat-saturated T2-weighted image and (f) sagittal contrast-enhanced spoiled gradient recalled echo fat-saturated T1-weighted image reveal the large soft tissue component within the quadriceps compartment that almost encircles the cortex. The cortex was affected only posteriorly, despite the large soft tissue mass $(e, f)$, whereas bone marrow involvement of the femur was best seen after gadolinium administration (f, arrows). 
a, b
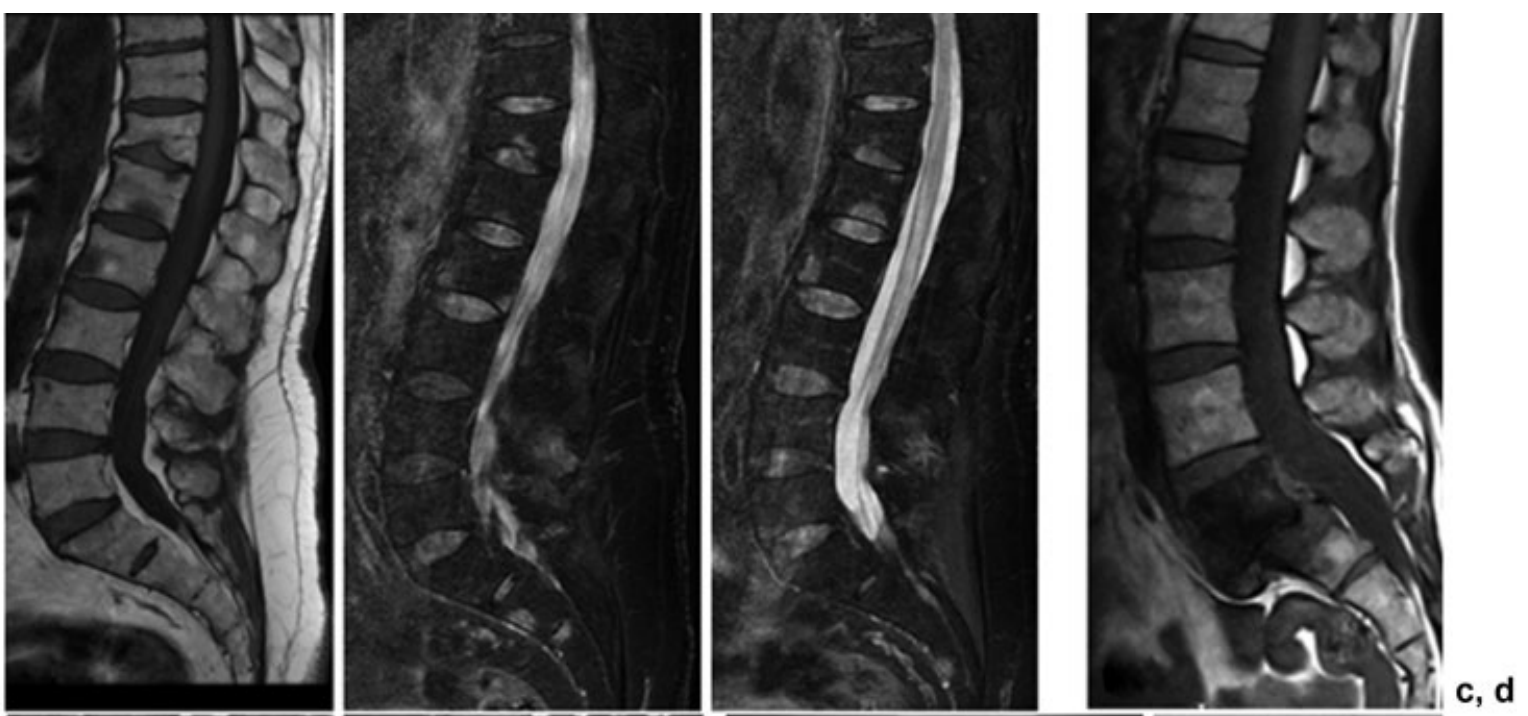

$e, f$
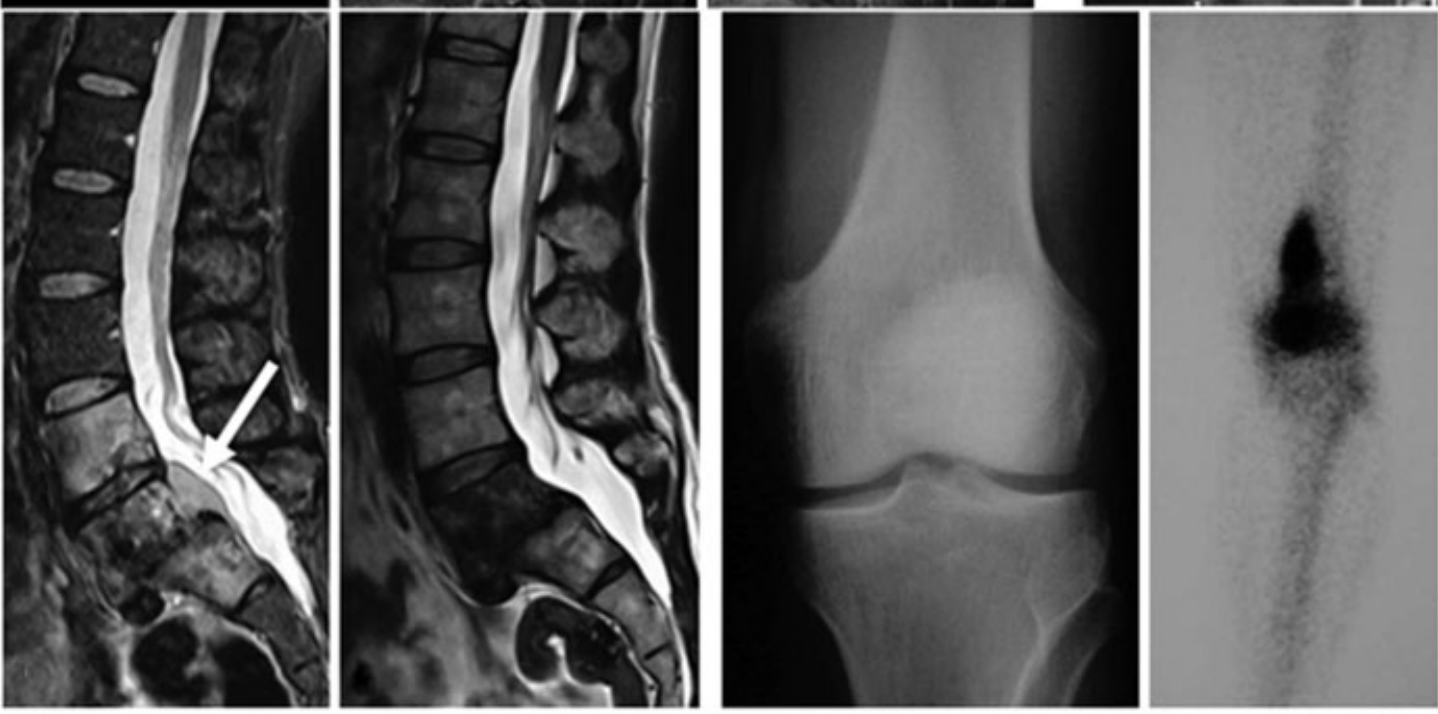

g, $\mathbf{h}$
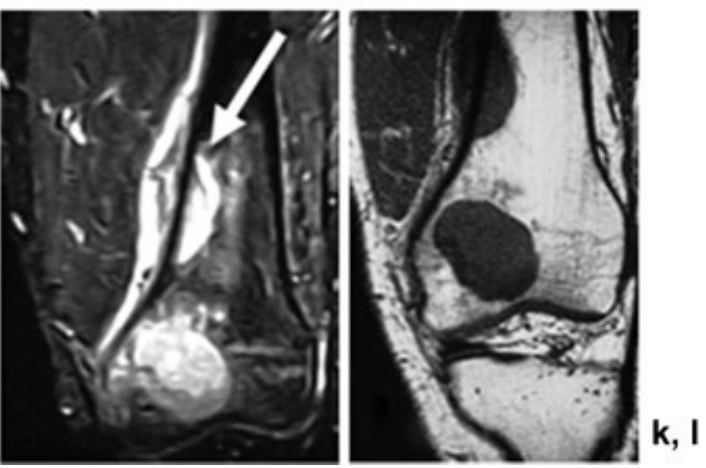

Fig. 8 Image gallery of bone lymphomas. (a-c) A 74-year-old man with osseous non-Hodgkin's lymphoma, initially misdiagnosed as seronegative spondylarthritis. (a) Sagittal T1-weighted and (b, c) sagittal proton-density-weighted fat-saturated sequences. The inferior anterior and posterior corners of $L 2$ are hypointense on T1-weighted image and hyperintense on fat-saturated T2-weighted image, respectively, whereas a lesion at the inferior end plate of L1 imitated an Andersson lesion. Repeat MRI after 3 months revealed more and larger focal lesions. (d-f) Multifocal non-Hodgkin's lymphoma in a 51-year-old woman presenting with intermittent back pain and infiltration of L4, L5, and S1 vertebral bodies as well as epidural infiltration at L5 level (arrow). (d) Sagittal T1-weighted, (e) sagittal short tau inversion recovery (STIR), and (f) sagittal T2-weighted sequences. Note the different signal behavior on T2-weighted images of the osseous manifestations. There is mixed signal intensity of L5 vertebral body on STIR image (e) and low signal intensity on T1-weighted and T2-weighted images; a mild hyperintensity of L4 and S1 is shown only on STIR MR images. (g-l) Non-Hodgkin's lymphoma of the right distal femur in a 39-year-old man with increasing pain in the left knee without a history of trauma. (g) Projection radiograph in anteroposterior view, (h) nuclear bone scan, CT in (i) coronal and (j) axial reformation, (k) MRI using coronal STIR, and (I) T1-weighted sequences. The radiograph of the left knee demonstrated a radiopaque lesion at the distal metadiaphyseal region of the left femur, with associated periosteal reaction, and the bone scintigraphy with technetium-99 MDP revealed intense radiotracer uptake. The coronal reformatted CT image shows in addition to (f) cortical destruction (arrows) along with one more lytic lesion in the epiphysis. The two lesions have low signal intensity on (I) T1-weighted image and high signal intensity on (k) STIR images. 

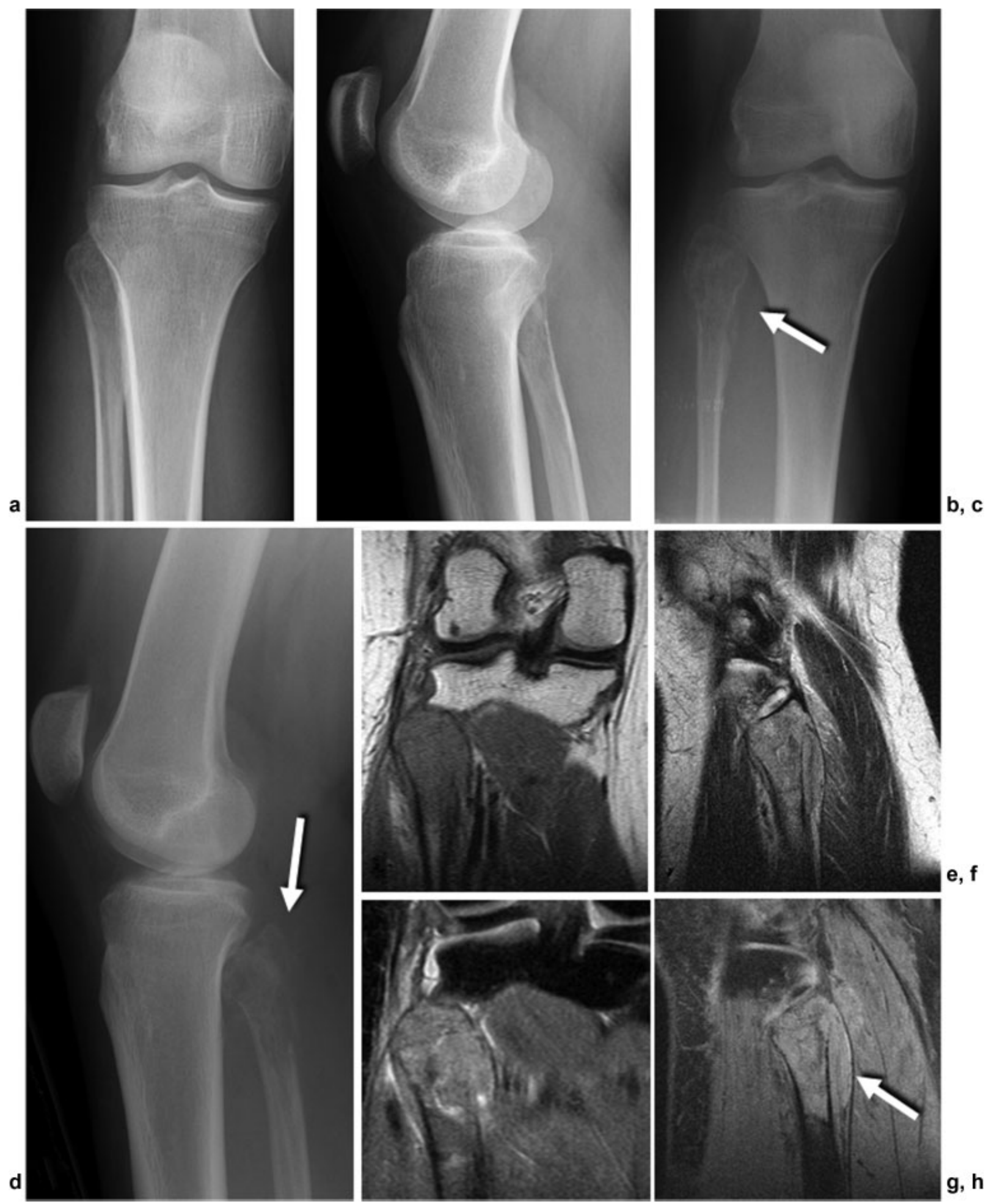

Fig. 9 Primary osseous B-cell non-Hodgkin's lymphoma in a 22-year-old man with painful swelling and tenderness of the right fibula head for 4 months, with no prior trauma and no fever. Projection radiography in (a) anteroposterior (AP) and (b) lateral view without obvious evidence of bony pathology. Projection radiography in (c) AP and (d) lateral view 6 weeks later clearly shows the destructive osteolysis of the fibular head (arrows). (e) MRI coronal T1-weighted, (f) sagittal T2-weighted, (g) coronal contrast-enhanced fat-saturated T1-weighted, (h) sagittal, (i) axial proton-density-weighted fat-saturated, and (j) axial contrast-enhanced fat-saturated T1-weighted sequences also illustrate the cortex exceeding infiltration and the wraparound sign (arrows). Staging was performed with scintigraphy (k) and contrast-enhanced CT (coronal reformation (I)) of the trunk, both showing no evidence of other skeletal or extraskeletal manifestations of lymphoma. Four months after six cycles of R-CHOP chemotherapy, whole-body MRI was performed for follow-up assessment $(\mathbf{m}, \mathbf{n})$ (coronal short tau inversion recovery), demonstrating a slight reduction of tumor size within the fibular head and no other manifestations of the lymphoma. Treatment was continued by radiotherapy with a total 32 Gy to the fibula. 

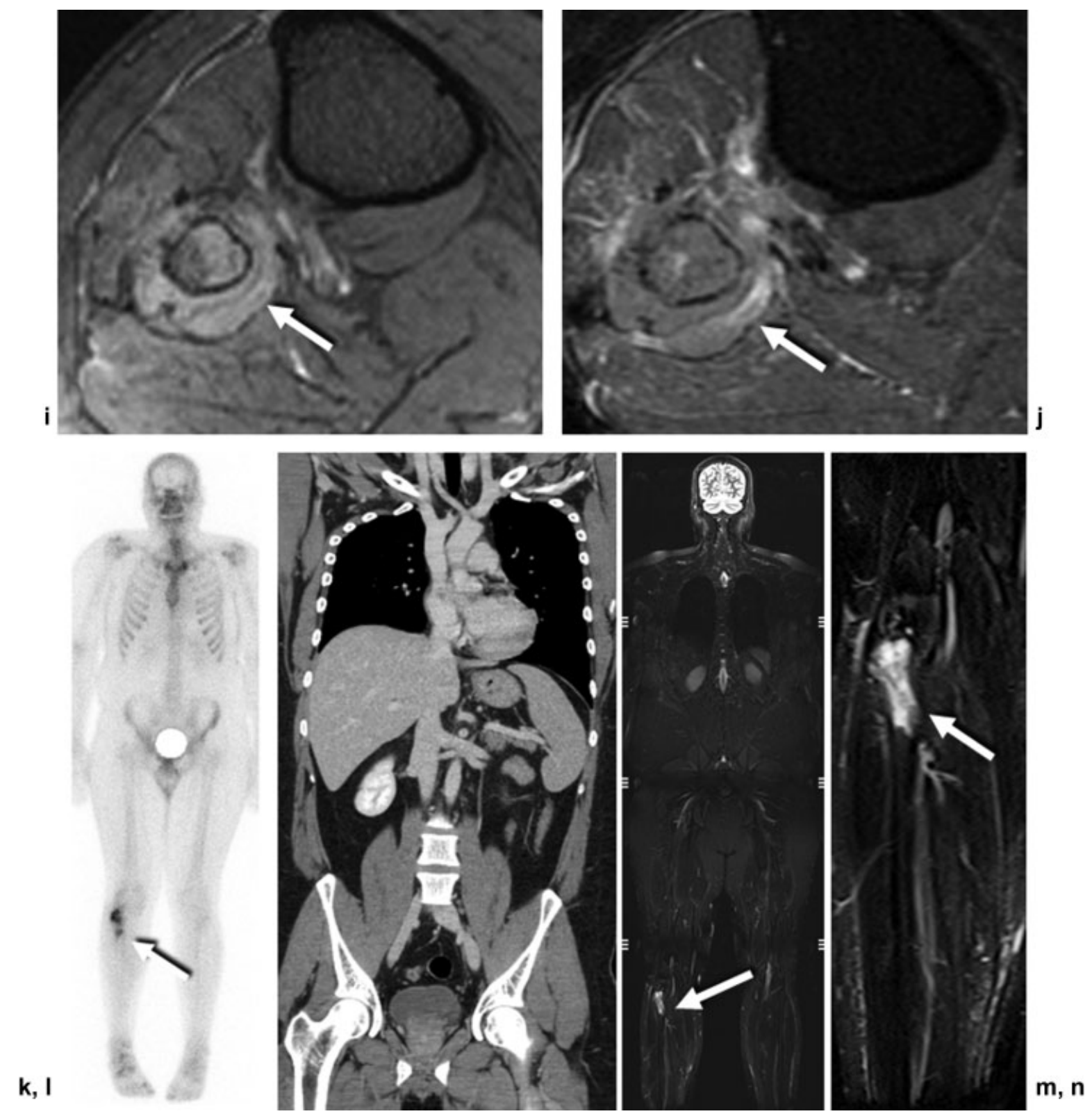

Fig. 9 (Continued)

spinal nerves. The signal behavior itself is nonspecific and may also show a diffuse or focal replacement of normal bone marrow, as could be seen in multiple myeloma (-Fig. 12).

Lymphoma of the deep soft tissues usually reveals long cones of intramuscular or intermuscular tumor, again best depicted by MRI. Cortical destruction allowing communication between the intraosseous and soft tissue components may be subtle with small striations of extension. ${ }^{36}$ Similar to ES, a wraparound sign may be observed consisting of an often large soft tissue component encasing the bone while the cortical bone appears preserved on MRI ( - Fig. 13). In reality, the tumor has penetrated the cortex in a permeative way.

Contrast-enhanced CT should be used for staging and detection of other organ manifestations (-Fig. 14). A bone scintigraphy may demonstrate the local osseous involvement and other bony manifestations of the lymphoma. The sensitivity to detect multifocal disease is lower than that of whole-body MRI. FDG-PET is well suitable for staging and treatment monitoring, but whole-body MRI (including diffusion-weighted imaging) may also be used for this purpose (-Fig.9); both have similar performance for the detection of bone marrow involvement. ${ }^{37,38}$

\section{Differential Diagnosis}

For differential diagnosis, the patient's age is the crucial parameter. Typical differential diagnoses in young patients include ES, unifocal Langerhans cell histiocytosis, and osteolytic osteosarcoma. In elderly patients, typical differential diagnoses comprise metastases (for instance of small-cell tumors like bronchial carcinoma), fibrosarcoma, and osteomyelitis. In aggressive osteomyelitis with a Lodwick grade II or III appearance, ${ }^{39,40}$ clinical presentation may favor 

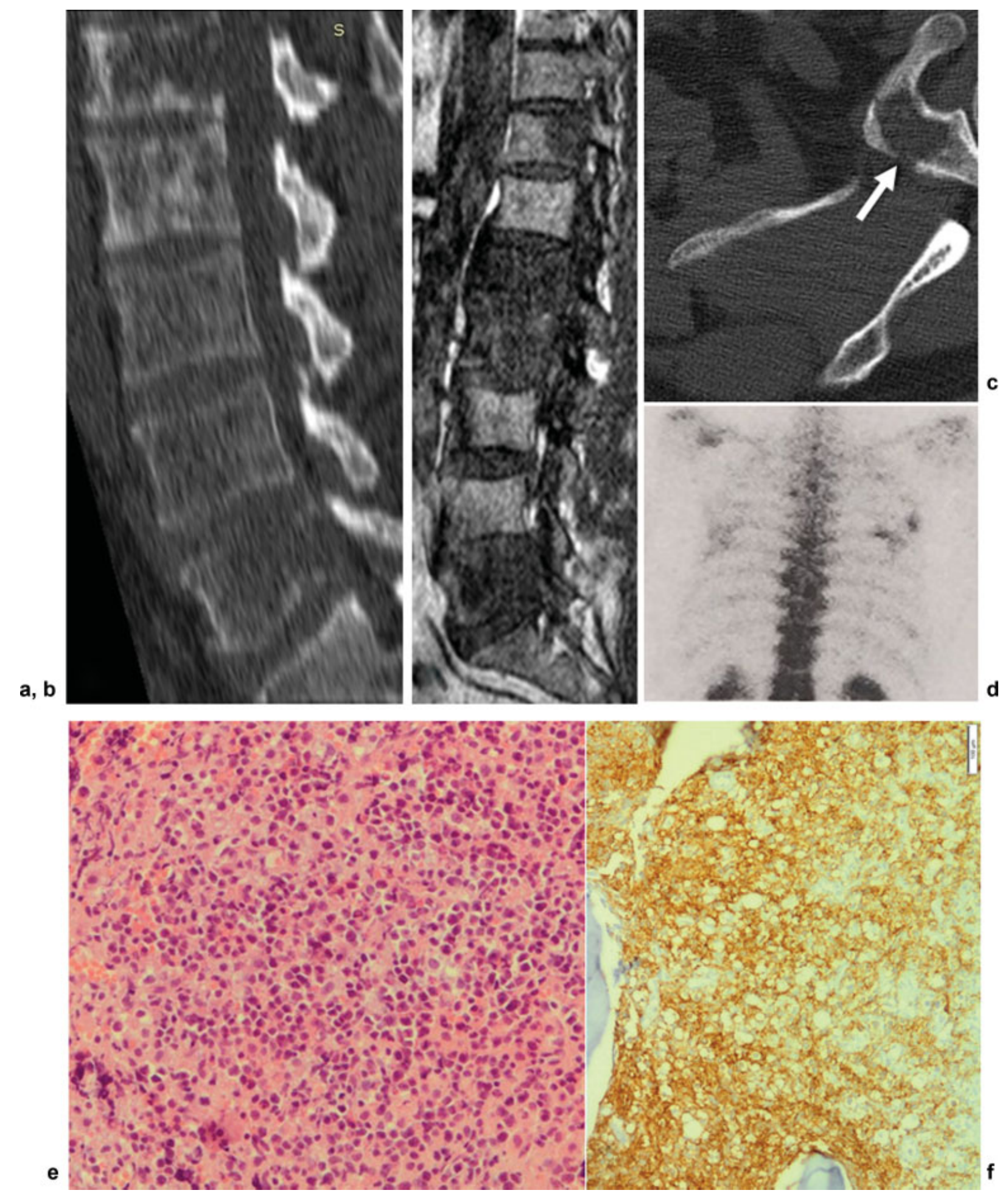

Fig. 10 Primary multifocal osseous diffuse large B-cell lymphoma in a 43-year-old man presenting with a 3-month history of back pain and pain of the left lower extremity. Hospitalization followed due to worsening of the symptoms and neurologic findings. (a) Sagittal CT and (b) sagittal T1weighted MRI of the lumbar spine illustrate several osseous predominantly lytic and marrow-replacing lesions located asymmetrically on vertebral bodies, pedicles, and several laminae. A well-defined glenoid lytic lesion is also present with small cortical disruption without a significant soft tissue mass (c, arrow on the chest CT). (d) Nuclear bone scan shows an intense tracer uptake of the vertebral column as well as some lesions within the ribs. There is no lymph node or visceral involvement. (e) Hematoxylin and eosin staining $(\times 100)$ illustrates that the tumor stroma consists of relatively monomorphous medium- to large-size cells and rare multinuclear cells, with hyperchromatic nuclei, prominent nucleoli, and scarce cytoplasm. (f) There also is diffuse homogeneous and strong CD20 immunostaining $(\times 200)$ supporting the diagnosis of diffuse large B-cell lymphoma. This case nicely illustrates the multifocal location on the spine with metastases as a typical differential diagnosis. 

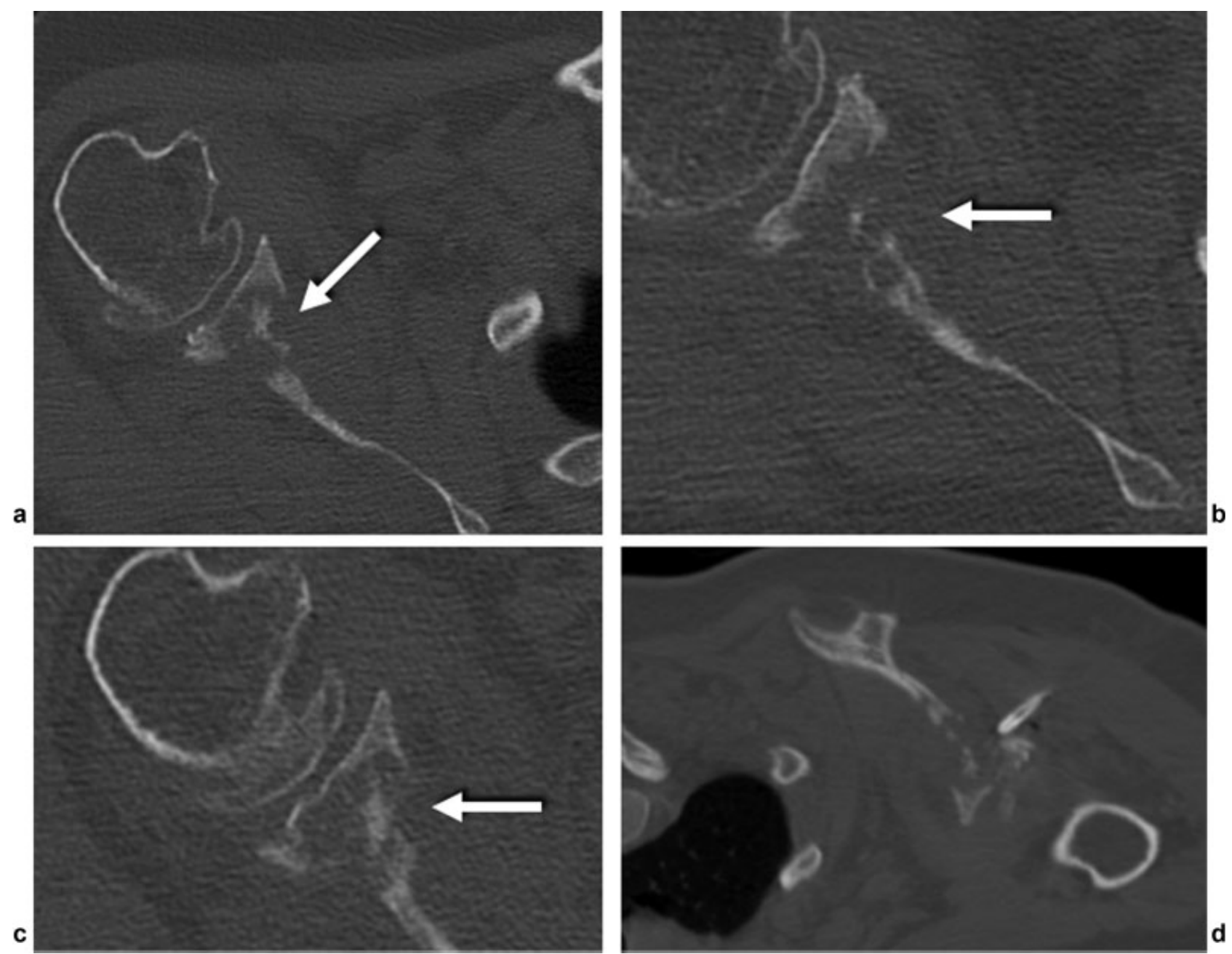

Fig. 11 (a-c) Non-Hodgkin's B-cell lymphoma in a 75-year-old woman presenting as both an osteolytic and osteosclerotic lesion of the glenoid in axial CT sections. The scapular lesion was an incidental finding. (d) CT-guided biopsy revealed an aggressive non-Hodgkin's diffuse large B-cell lymphoma.

inflammation. For confirmation of the final diagnosis, biopsy sampling and imaging correlation is always mandatory (-Figs. 13, 15, and 16).

\section{Therapy and Treatment Monitoring}

The mainstay of treatment is polychemotherapy ( - Fig. 13) and radiotherapy. ${ }^{24,31}$ Surgery is only indicated in case of complications, such as for instance in the case of pathologic fractures ${ }^{41}$ or spinal cord compression with neurologic deficits. The most common chemotherapy regimen for aggressive non-Hodgkin's lymphoma is cyclophosphamide, hydroxydaunorubicin (doxorubicin), oncovin (vincristine), and prednisone/prednisolone (CHOP). It was recently reported that primary osseous DLBCL had a 3 - and 5-year progression-free survival of $61.2 \%$ and $46.9 \%$, respectively, and 5- and 10 -year overall survival of $81.1 \%$ and $74.7 \%$, respectively. Multivariate analysis identified soft tissue extension and the International Prognostic Index score as the most important unfavorable prognostic factors. ${ }^{29}$ Also, multifocality was also significantly associated with a worse progression-free survival and overall survival, and the authors concluded that multifocal bone involvement is more similar to secondary bone lymphoma in characteristics and survival rather than unifocal bone disease, and thus it should be better classified and treated similar to the secondary bone lymphomas. ${ }^{42}$ Extraskeletal involvement and older age are adverse prognostic factors. For systemic lymphoma with bone involvement, disease-free 5 -year survival is $44 \% .^{25,43}$

\section{Conclusion}

The typical manifestation of ES is a permeative lesion in the diaphysis of a long bone in a child. However, the imaging appearance in projection radiography may be diverse, and ES has been called the chameleon of the bone tumors. Extraskeletal ES may be missed on CR and will best be depicted by MRI. There is no single characteristic imaging appearance of an osseous lymphoma. An osseous lymphoma should be considered in the differential diagnosis when encountering in an adult a Lodwick grade II or III osteolytic lesion in the metaphysis or diaphysis of a large long bone, the pelvis, or the vertebral column that may be accompanied to a greater or lesser extent by sclerotic bony changes. Similar to ES, PLB may also be called the chameleon of the bone tumors. In general, permeative and moth-eaten destructive patterns of the bone 


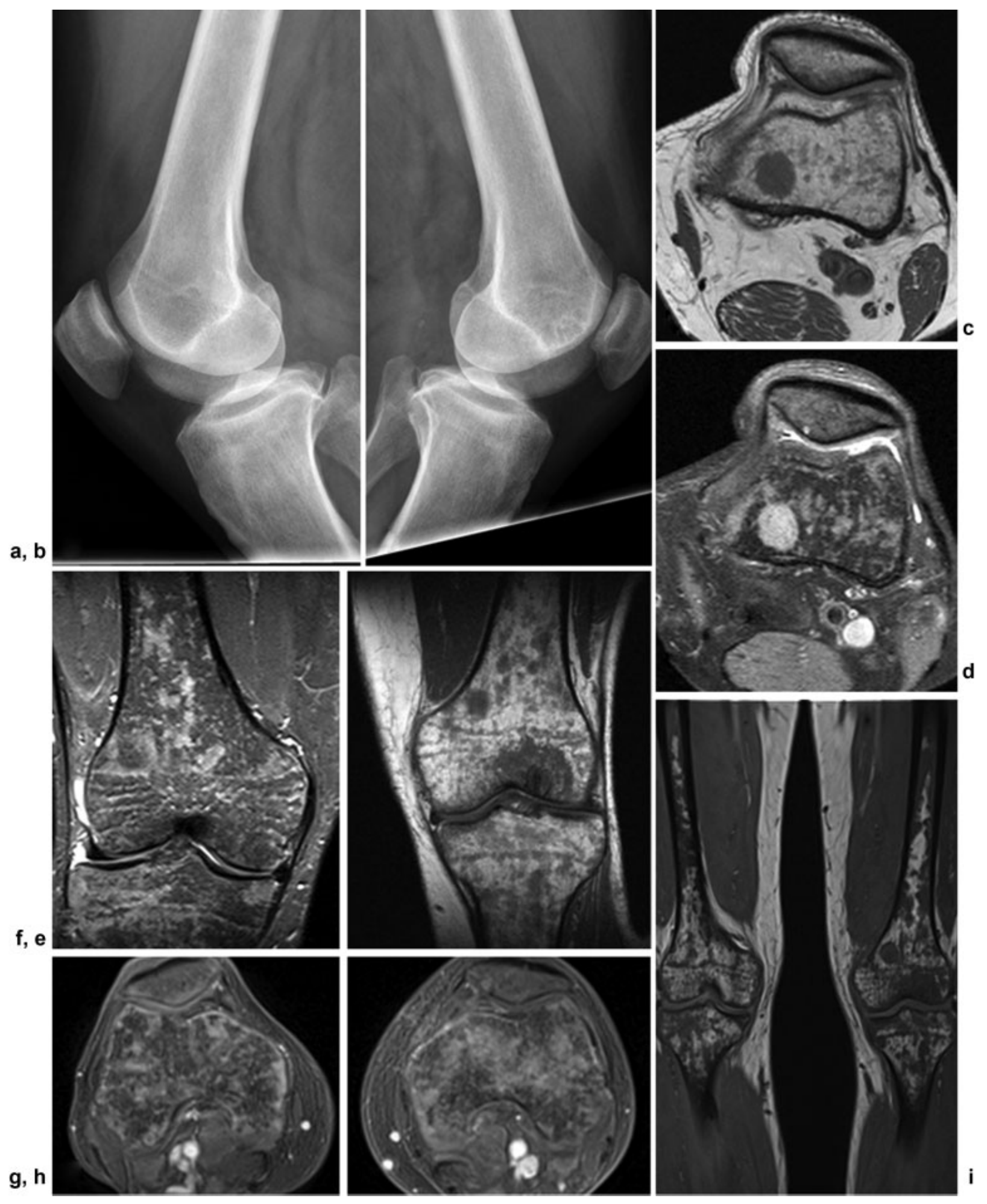

Fig. 12 Indolent non-Hodgkin's B-cell lymphoma in a 69-year-old man. Pain in both knees for 1 year was most pronounced around the right knee, with no B symptoms. Projection radiographs in lateral views, (a) right knee, (b) left knee, reveal no obvious bony destruction. (c) MRI axial T1weighted, (d) axial fat-saturated proton-density-weighted, (e) coronal T1-weighted sequence on the left side, and (f) coronal short tau inversion recovery sequence on the right side reveal multifocal lymphomatous bone marrow infiltration confirmed at histology. (g) MRI axial contrastenhanced T1-weighted fat-saturated sequence on the right side and (h) on the left side, and (i) coronal T1-weighted sequence performed 4 months after first MRI shows an increase of the diffuse lymphomatous infiltration of the bone marrow but no extraosseous manifestation, as confirmed by contrast-enhanced CT of the trunk (not shown). 
a
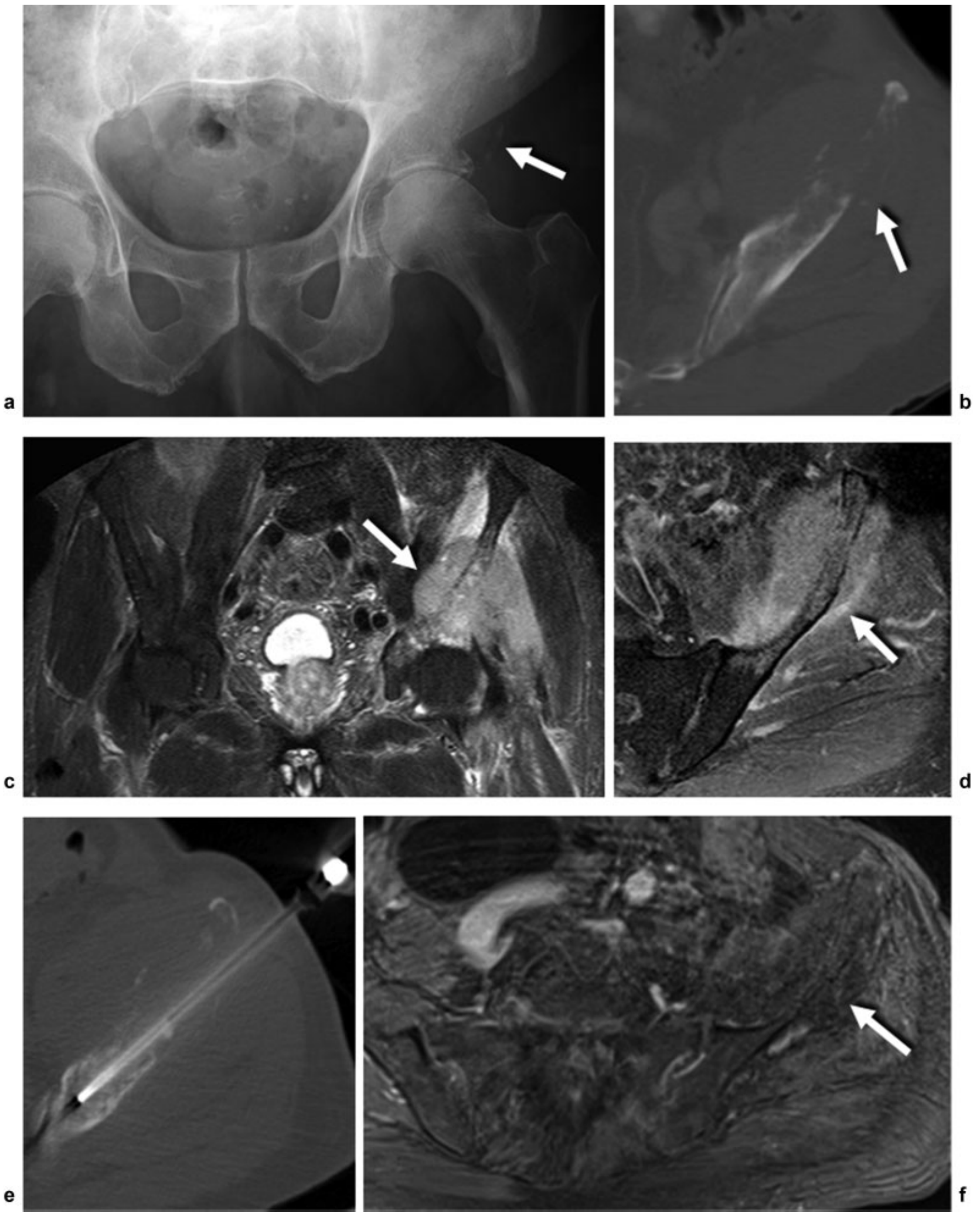

Fig. 13 Non-Hodgkin's B-cell lymphoma in an 82-year-old man. (a) Projection radiograph of the pelvis was performed because of increasing pain in the left groin for 3 weeks, weight loss, and B symptoms, as well as a locally palpable lump. (b) Axial CT, (c) coronal short tau inversion recovery, and (d) axial contrast-enhanced fat-saturated T1-weighted sequence. The osteolysis of the iliac bone with partly calcified periosteal shell (arrows in [a] and [b]) presented at MRI with a large and homogeneous soft tissue component that penetrated the cortical bone (arrows in [c] and [d]). A wraparound sign is also present. (e) CT-guided biopsy revealed an aggressive non-Hodgkin's diffuse large B-cell lymphoma. (f) The axial contrast-enhanced fat-saturated T1-weighted sequence performed after six cycles of R-CHOP chemotherapy clearly demonstrates treatment response with nearly absent contrast enhancement and a nearly vanished soft tissue component (arrow). 

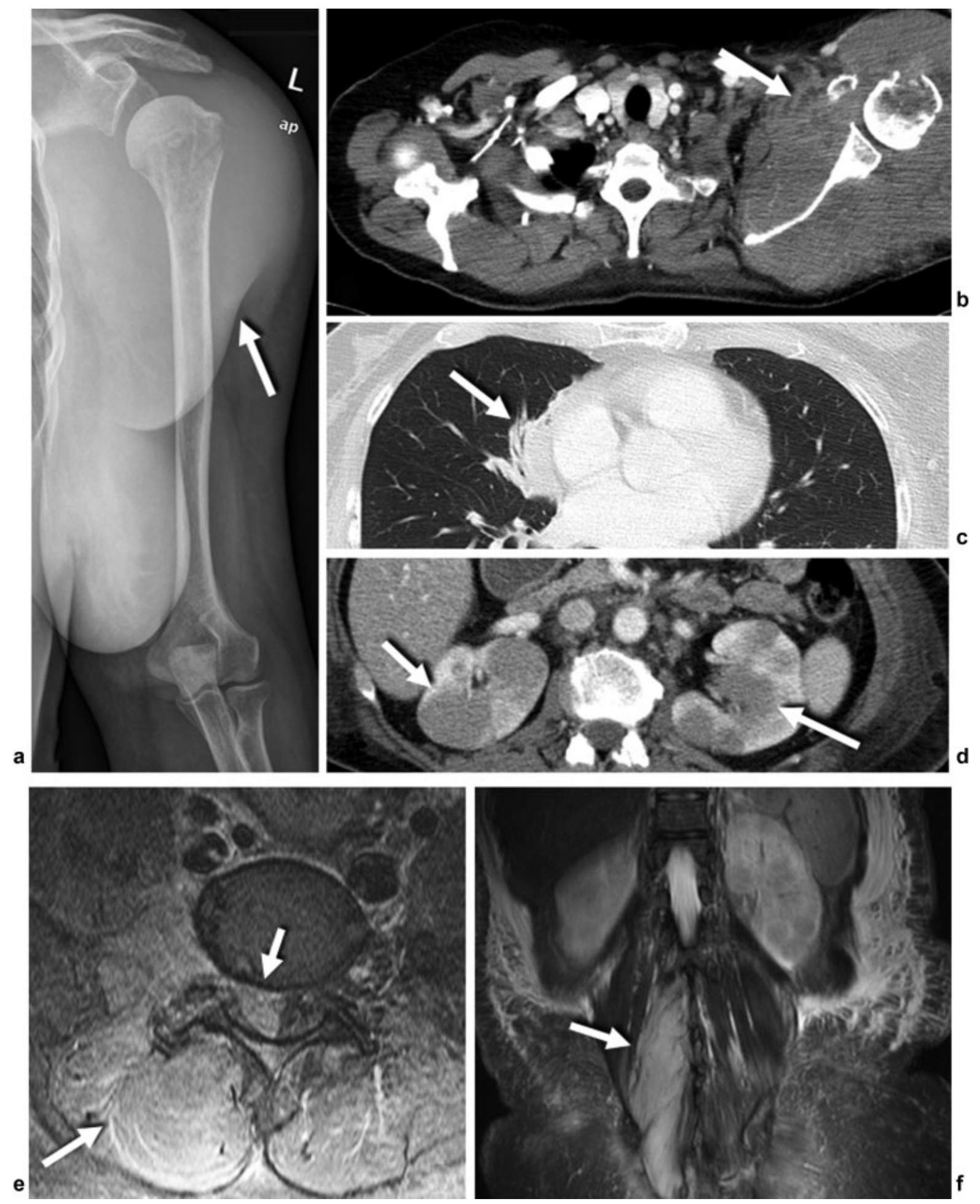

Fig. 14 Non-Hodgkin's diffuse large B-cell lymphoma in a 59-year-old woman with a multiorgan manifestation (stage IV). The left shoulder joint and upper arm were first to present with symptoms (swelling for 2 weeks, no pain medication). (a) Projection radiograph shows soft tissue opacification around the left shoulder. (b) Contrast-enhanced staging CT revealed large mass with enhancement of the left shoulder joint and scapula as well as infiltration of the anterior humerus (b), lung (c, arrow), and bilateral kidney infiltration visible as multiple hypodense lesions with blurred border (d, arrows), infiltration of the spinal canal and erector spinae muscle (e, arrows). Axial contrast-enhanced fat-saturated T1-weighted and (f) coronal short tau inversion recovery sequence. 
a, b
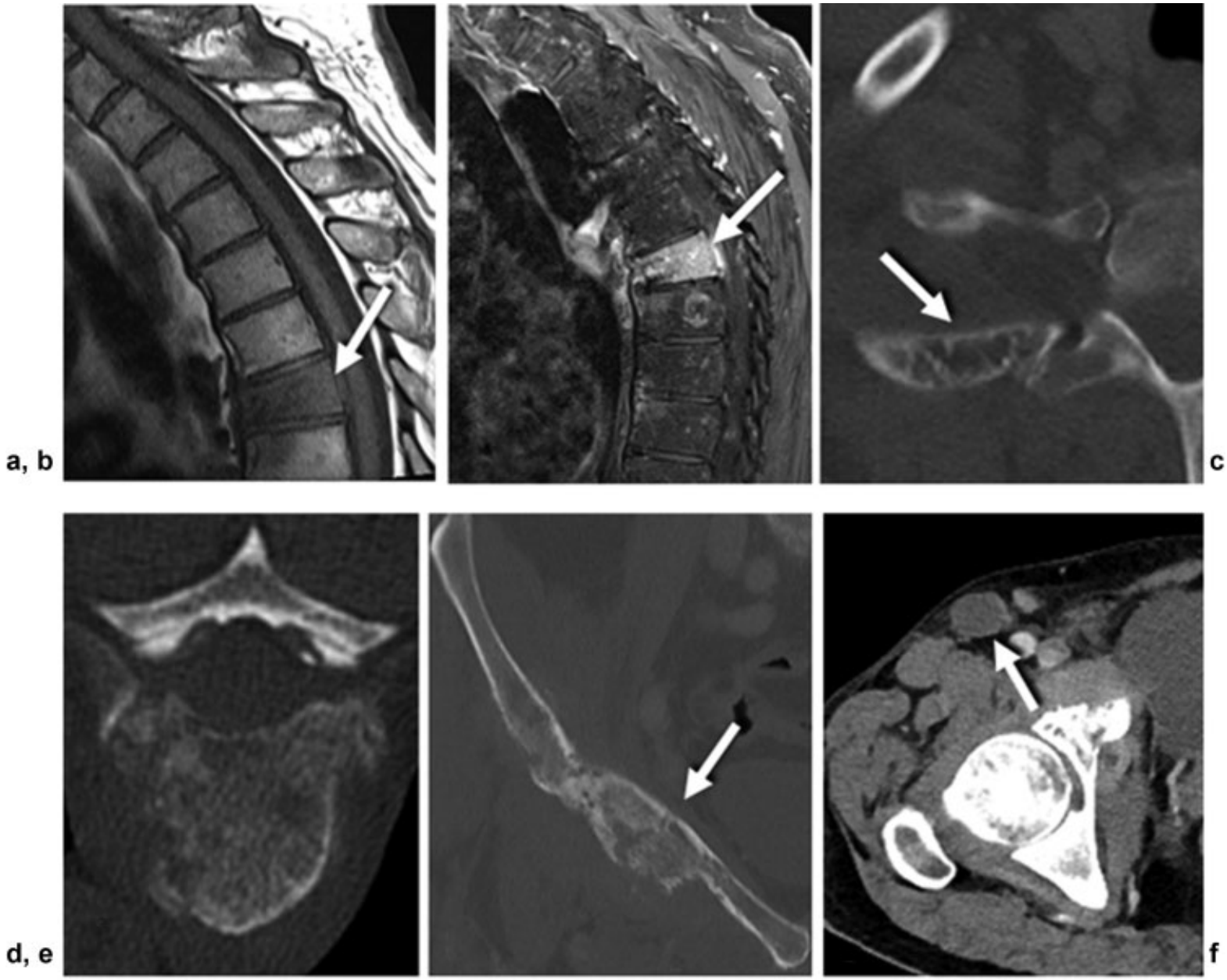

Fig. 15 Non-Hodgkin's B-cell lymphoma in a 69-year-old man with multifocal osseous manifestations. First symptom was an episode of sudden back pain without trauma lasting for 2 months. (a) MRI sagittal T1-weighted and (b) contrast-enhanced fat-saturated T1-weighted sequences demonstrated infiltration of the bone marrow of the sixth thoracic vertebra (arrows) and a slightly wedge-shaped vertebra. (c) Staging CT of the trunk revealed osseous lesions in the second rib of the right side (arrow), (d) the sixth thoracic vertebra, and (e) the acetabulum (arrow) and os pubis (not shown), as well as (f) an enlarged lymph node within the right groin (arrow) and no other enlarged lymph nodes. (g) CT-guided biopsy of the sixth thoracic vertebra was performed, and histology revealed diffuse large B-cell lymphoma. 

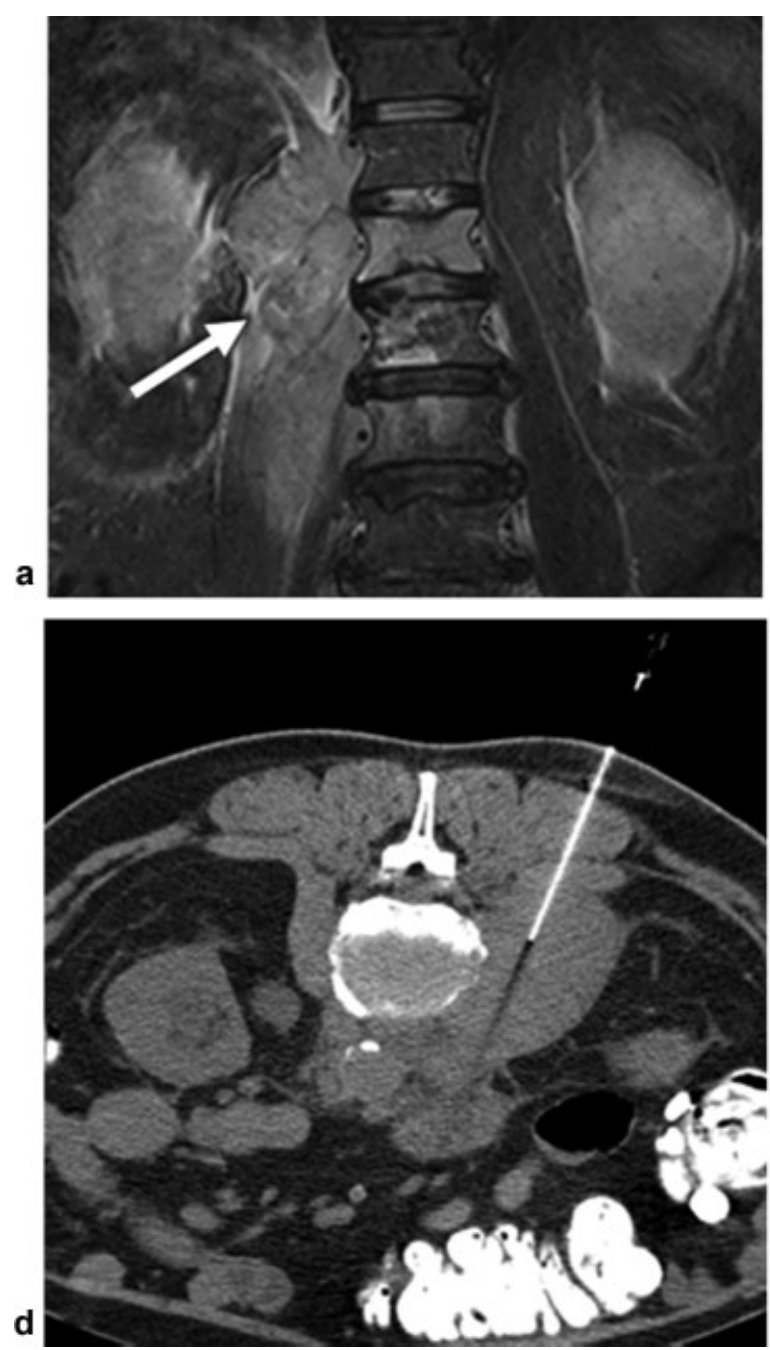

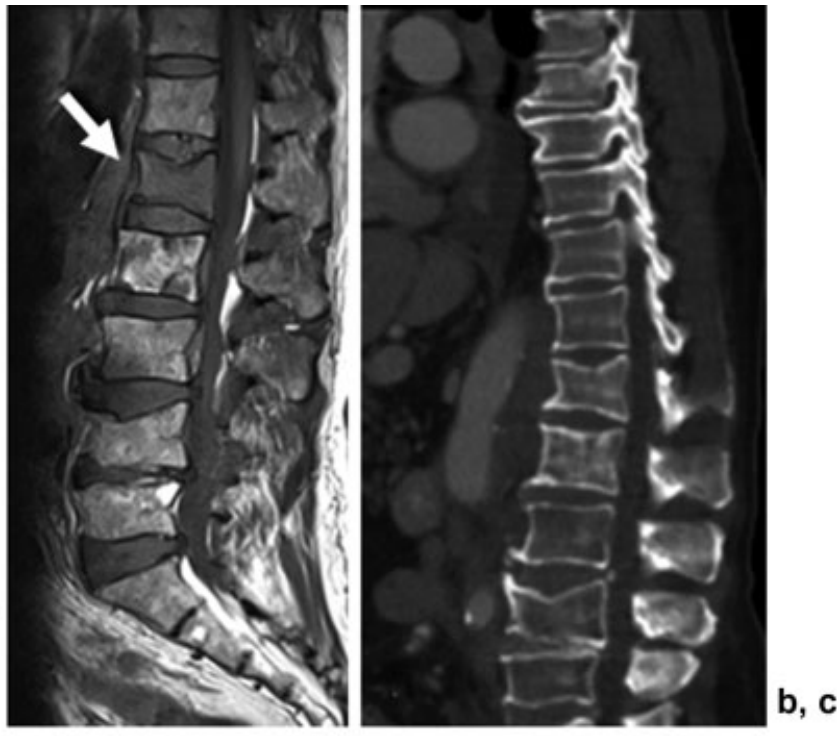

Fig. 16 Aggressive non-Hodgkin's diffuse large B-cell lymphoma at stage IV in a 76-year-old man with severe back pain and weight loss for 2 months. (a) MRI coronal short tau inversion recovery and (b) sagittal T1-weighted sequence revealed infiltration of first lumbar vertebra (arrow) and large paravertebral mass next to the right psoas muscle (arrow). (c) CT in sagittal reformation and (d) CT-guided biopsy for histologic and immunohistochemical verification.

are a hint for a small- or round-cell tumor. Histologic confirmation should always be sought. Because patients with remarkably normal-appearing CR may show distinct abnormalities on MRI or bone scintigraphy, in patients with persisting symptoms but negative $\mathrm{CR}$, further assessment with a more sensitive modality, such as MRI, is essential.

\section{References}

1 Freyschmidt J, Ostertag H. Ewing's sarcoma, fibrogenic tumors, giant cell tumor, hemangioma of bone : radiology and pathology [in German]. Radiologe 2016;56(06):520-535

2 Peersman B, Vanhoenacker FM, Heyman S, et al. Ewing's sarcoma: imaging features. JBR-BTR 2007;90(05):368-376

3 Jordanov MI, Block JJ, Gonzalez AL, Green NE. Transarticular spread of Ewing sarcoma mimicking septic arthritis. Pediatr Radiol 2009;39(04):381-384

4 Ilaslan H, Sundaram M, Unni KK, Dekutoski MB. Primary Ewing's sarcoma of the vertebral column. Skeletal Radiol 2004;33(09): 506-513
5 Rodallec MH, Feydy A, Larousserie F, et al. Diagnostic imaging of solitary tumors of the spine: what to do and say. Radiographics 2008;28(04):1019-1041

6 Delattre 0, Zucman J, Melot T, et al. The Ewing family of tumors-a subgroup of small-round-cell tumors defined by specific chimeric transcripts. N Engl J Med 1994;331(05):294-299

7 Lessnick SL, Ladanyi M. Molecular pathogenesis of Ewing sarcoma: new therapeutic and transcriptional targets. Annu Rev Pathol 2012;7:145-159

8 Murphey MD, Senchak LT, Mambalam PK, Logie CI, KlassenFischer MK, Kransdorf MJ. From the radiologic pathology archives: Ewing sarcoma family of tumors: radiologic-pathologic correlation. Radiographics 2013;33(03):803-831

9 Applebaum MA, Goldsby R, Neuhaus J, DuBois SG. Clinical features and outcomes in patients with Ewing sarcoma and regional lymph node involvement. Pediatr Blood Cancer 2012;59(04):617-620

10 Study in localized and disseminated Ewing sarcoma. https://clinicaltrials.gov/ct2/show/NCT00987636. Accessed September 2, 2018

11 Lalam R, Bloem JL, Noebauer-Huhmann IM, et al. ESSR consensus document for detection, characterization, and referral pathway for tumors and tumorlike lesions of bone. Semin Musculoskelet Radiol 2017;21(05):630-647 
12 McCarville MB, Chen JY, Coleman JL, et al. Distinguishing osteomyelitis from Ewing sarcoma on radiography and MRI. AJR Am J Roentgenol 2015;205(03):640-650; quiz 651

13 Kloth JK, Wolf M, Rehnitz C, Lehner B, Wiedenhöfer B, Weber MA. Radiological diagnostics of spinal tumors. Part 1: general tumor diagnostics and special diagnostics of extradural tumors [in German]. Orthopade 2012;41(08):595-607

14 Haeusler J, Ranft A, Boelling T, et al. The value of local treatment in patients with primary, disseminated, multifocal Ewing sarcoma (PDMES). Cancer 2010;116(02):443-450

15 Potratz J, Dirksen U, Jürgens H, Craft A. Ewing sarcoma: clinical state-of-the-art. Pediatr Hematol Oncol 2012;29(01):1-11

16 Ladenstein R, Pötschger U, Le Deley MC, et al. Primary disseminated multifocal Ewing sarcoma: results of the Euro-EWING 99 trial. J Clin Oncol 2010;28(20):3284-3291

17 AWMF guideline to Ewing's sarcoma (in children and adolescents) of the German Society of Pediatric and Adolescent Medicine (DGKJ). In: AWMF online (state 2014, valid until June 1, 2019). https://www.awmf.org/uploads/tx_szleitlinien/0250061_S1_Ewing_Sarkome_Kinder_Jugendliche_2014-06.pdf. Accessed August 12, 2018

18 Vilanova JC, Baleato-Gonzalez S, Romero MJ, Carrascoso-Arranz J, Luna A. Assessment of musculoskeletal malignancies with functional MR imaging. Magn Reson Imaging Clin N Am 2016;24(01):239-259

19 Paulussen M, Bielack S, Jürgens H, Casali PG; ESMO Guidelines Working Group. Ewing's sarcoma of the bone: ESMO clinical recommendations for diagnosis, treatment and follow-up. Ann Oncol 2009;20(Suppl 4):140-142

20 Balamuth NJ, Womer RB. Ewing's sarcoma. Lancet Oncol 2010;11 (02):184-192

21 Moore DD, Haydon RC. Ewing's sarcoma of bone. Cancer Treat Res 2014;162:93-115

22 Binesh F, Mirjalili MR, Akhavan A, Navabii H. Primary bony Hodgkin's lymphoma. BMJ Case Rep 2012;2012;

23 Messina C, Christie D, Zucca E, Gospodarowicz M, Ferreri AJ. Primary and secondary bone lymphomas. Cancer Treat Rev 2015;41(03):235-246

24 Ludwig K. Musculoskeletal lymphomas [in German]. Radiologe 2002;42(12):988-992

25 Demircay E, Hornicek FJ Jr, Mankin HJ, Degroot H III. Malignant lymphoma of bone: a review of 119 patients. Clin Orthop Relat Res 2013;471(08):2684-2690

26 Jain A, Alam K, Maheshwari V, Khan R, Nobin H, Narula V. Primary bone lymphomas-clinical cases and review of literature. J Bone Oncol 2013;2(03):132-136

27 Chisholm KM, Ohgami RS, Tan B, Hasserjian RP, Weinberg OK. Primary lymphoma of bone in the pediatric and young adult population. Hum Pathol 2017;60:1-10
28 Fox MG, Marti JK, Bachmann KR, LeGallo RD, Foster WC. Epiphyseal presentation of non-Hodgkin's lymphoma of bone in two pediatric patients-one with primary lymphoma of bone. Skeletal Radiol 2015;44(04):587-595

$29 \mathrm{Wu} \mathrm{H}$, Zhang L, Shao H, et al. Prognostic significance of soft tissue extension, international prognostic index, and multifocality in primary bone lymphoma: a single institutional experience. $\mathrm{Br} \mathrm{J}$ Haematol 2014;166(01):60-68

30 Navarro SM, Matcuk GR, Patel DB, et al. Musculoskeletal imaging findings of hematologic malignancies. Radiographics 2017;37 (03):881-900

31 Krishnan A, Shirkhoda A, Tehranzadeh J, Armin AR, Irwin R, Les K. Primary bone lymphoma: radiographic-MR imaging correlation. Radiographics 2003;23(06):1371-1383; discussion 1384-1387

32 Ruzek KA, Wenger DE. The multiple faces of lymphoma of the musculoskeletal system. Skeletal Radiol 2004;33(01):1-8

33 Graham TS. The ivory vertebra sign. Radiology 2005;235(02): 614-615

34 Braun RA, Milito CF, Goldman SM, Fernandes EdeÁ. Ivory vertebra: imaging findings in different diagnoses. Radiol Bras 2016;49 (02):117-121

35 Lim CY, Ong KO. Imaging of musculoskeletal lymphoma. Cancer Imaging 2013;13(04):448-457

36 Murphey MD, Kransdorf MJ. Primary musculoskeletal lymphoma. Radiol Clin North Am 2016;54(04):785-795

37 Albano D, Patti C, Lagalla R, Midiri M, Galia M. Whole-body MRI, FDG-PET/CT, and bone marrow biopsy, for the assessment of bone marrow involvement in patients with newly diagnosed lymphoma. J Magn Reson Imaging 2017;45(04):1082-1089

38 Balbo-Mussetto A, Saviolo C, Fornari A, et al. Whole body MRI with qualitative and quantitative analysis of DWI for assessment of bone marrow involvement in lymphoma. Radiol Med (Torino) 2017;122(08):623-632

39 Lodwick GS, Wilson AJ, Farrell C, Virtama P, Dittrich F. Determining growth rates of focal lesions of bone from radiographs. Radiology 1980;134(03):577-583

40 Lodwick GS, Wilson AJ, Farrell C, Virtama P, Smeltzer FM, Dittrich F. Estimating rate of growth in bone lesions: observer performance and error. Radiology 1980;134(03):585-590

41 Siddiqui YS, Khan AQ, Sherwani M. Pathological fractures in primary non-Hodgkin's lymphoma of the bone: a case series with review of the literature. J Clin Diagn Res 2013;7(03):513-517

$42 \mathrm{Wu} \mathrm{H}$, Bui MM, Leston DG, et al. Clinical characteristics and prognostic factors of bone lymphomas: focus on the clinical significance of multifocal bone involvement by primary bone large B-cell lymphomas. BMC Cancer 2014;14:900

43 Dürr HR, Müller PE, Hiller E, et al. Malignant lymphoma of bone. Arch Orthop Trauma Surg 2002;122(01):10-16 